Louisiana State University

LSU Digital Commons

Faculty Publications

Department of Biological Sciences

$10-1-2019$

\title{
Mechanistic target of rapamycin (mTOR) implicated in plasticity of the reproductive axis during social status transitions
}

\author{
Karen P. Maruska \\ Louisiana State University \\ Young Chang Sohn \\ Gangneung-Wonju National University \\ Russell D. Fernald \\ Stanford University
}

Follow this and additional works at: https://digitalcommons.Isu.edu/biosci_pubs

\section{Recommended Citation}

Maruska, K., Sohn, Y., \& Fernald, R. (2019). Mechanistic target of rapamycin (mTOR) implicated in plasticity of the reproductive axis during social status transitions. General and Comparative Endocrinology, 282 https://doi.org/10.1016/j.ygcen.2019.113209

This Article is brought to you for free and open access by the Department of Biological Sciences at LSU Digital Commons. It has been accepted for inclusion in Faculty Publications by an authorized administrator of LSU Digital Commons. For more information, please contact ir@lsu.edu. 


\title{
Mechanistic target of rapamycin (mTOR) implicated in plasticity of the reproductive axis during social status transitions
}

\author{
Karen P. Maruska ${ }^{\mathrm{a}, *}$, Young Chang Sohn ${ }^{\mathrm{b}}$, Russell D. Fernald ${ }^{\mathrm{c}}$ \\ ${ }^{a}$ Department of Biological Sciences, Louisiana State University, Baton Rouge, LA 70803, United States \\ ${ }^{\mathrm{b}}$ Department of Marine Molecular Bioscience, Gangneung-Wonju National University, Gangneung, Gangwon 25457, Republic of Korea \\ ${ }^{\mathrm{c}}$ Department of Biology, Stanford University, Stanford, CA 94305, United States
}

\section{A R T I C L E I N F O}

\section{Keywords:}

Brain

Cichlid

GnRH1

Pituitary

Teleost

Testis

\begin{abstract}
A B S T R A C T
The highly conserved brain-pituitary-gonadal (BPG) axis controls reproduction in all vertebrates, so analyzing the regulation of this signaling cascade is important for understanding reproductive competence. The protein kinase mechanistic target of rapamycin (mTOR) functions as a conserved regulator of cellular growth and metabolism in all eukaryotes, and also regulates the reproductive axis in mammals. However, whether mTOR might also regulate the BPG axis in non-mammalian vertebrates remains unexplored. We used complementary experimental approaches in an African cichlid fish, Astatotilapia burtoni, to demonstrate that mTOR is involved in regulation of the brain, pituitary, and testes when males rise in rank to social dominance. mTOR or downstream components of its signaling pathway (p-p70S6K) were detected in gonadotropin-releasing hormone (GnRH1) neurons, the pituitary, and testes. Transcript levels of mtor in the pituitary and testes also varied when reproductively-suppressed subordinate males rose in social rank to become dominant reproductively-active males, a transition similar to puberty in mammals. Intracerebroventricular injection of the mTORC1 inhibitor, rapamycin, revealed a role for mTOR in the socially-induced hypertrophy of GnRH1 neurons. Rapamycin treatment also had effects at the pituitary and testes, suggesting involvement of the mTORC1 complex at multiple levels of the reproductive axis. Thus, we show that mTOR regulation of BPG function is conserved to fishes, likely playing important roles in regulating reproduction and fertility across all male vertebrates.
\end{abstract}

\section{Introduction}

Neuronal plasticity enables the nervous system to adapt to physical and social challenges, and identifying the mechanisms responsible for such plasticity is crucial for understanding dynamic brain functions. One critical brain function is the control of reproduction, which can be influenced by both internal (e.g. metabolic, hormonal) and external (e.g. environmental, social) cues. Reproduction in all vertebrates is regulated via the highly conserved brain-pituitary-gonadal (BPG) axis. At the apex of this axis are the gonadotropin-releasing hormone 1 (GnRH1)-containing neurons in the brain that integrate external and internal information, and deliver GnRH1 to the pituitary where it initiates a signaling cascade to modulate reproductive behavior and physiology. These GnRH1 neurons exhibit dramatic cellular and molecular plasticity across vertebrate taxa [fish: (Davis and Fernald, 1990; Ma et al., 2015; Maruska, 2015), amphibians: (Burmeister and Wilczynski, 2005), birds: (MacDougall-Shackleton et al., 2009; Stevenson et al., 2012), and mammals: (Kriegsfeld and Nelson, 1999;
Serra et al., 2017)], serving to regulate seasonal, metabolic, and social changes in reproductive capacity and fertility (Sucularli et al., 2016). Social signals from conspecifics in particular may be a driving force for reproductive plasticity, which is ultimately mediated by the GnRH1 neurons. For example, the number, size, and morphology of GnRH1 neurons can be influenced by the social environment in fish, frogs, birds, and mammals (Burmeister and Wilczynski, 2005; Davis and Fernald, 1990; Dellovade and Rissman, 1994; Sucularli et al., 2016). These changes in GnRH1 neuron activity and function cause plastic changes along the reproductive axis, including variations in behavior as well as in the pituitary (e.g. release of luteinizing hormone, LH, and follicle stimulating hormone, FSH) and in the gonads (e.g. gametogenesis, steroid production). GnRH1 neurons and the pituitary are also regulated by a negative feedback loop from sex-steroid hormones produced by the gonads. Thus, while there is clear evidence for reproductive plasticity associated with social signals across taxa, the exact cellular signaling mechanisms remain unresolved, particularly in non-mammalian vertebrates.

\footnotetext{
* Corresponding author.

E-mail address: kmaruska@1su.edu (K.P. Maruska).
} 
The African cichlid fish Astatotilapia burtoni is a well-suited model to identify the mechanisms responsible for socially-induced neuronal and reproductive plasticity (Maruska et al., 2011). Males exist as dominant or subordinate phenotypes, and can rapidly and reversibly switch between these social ranks in response to social or environmental disturbance. Since dominant males aggressively defend and maintain territories, they also have an up-regulated reproductive axis and more opportunities to spawn with females [reviewed in (Fernald and Maruska, 2012; Maruska, 2014; Maruska et al., 2014; Maruska and Fernald, 2018). When a subordinate male has the opportunity to rise in social rank and become a dominant territory-holding male, there are rapid (minutes) and dramatic changes in behavior and coloration, as well as many physiological changes along the reproductive axis from the brain to the testes that occur within minutes to days (Burmeister et al., 2005; Maruska and Fernald, 2010; Maruska and Fernald, 2011; Maruska et al., 2007). One of the most striking and important changes seen during the male transition to social dominance in A. burtoni is an 8fold enlargement in volume of GnRH1 neurons in the preoptic area, and a corresponding increased complexity of their dendritic arbors (Davis and Fernald, 1990; Francis et al., 1993; Schell et al., 2016). These morphological changes also have profound effects on the functional output of GnRH1 excitable cells, including neuron firing synchronization and increased GnRH1 production, which in turn influences reproductive physiology, specifically through remodeling the connections among GnRH1 neurons (Greenwood and Fernald, 2004; Ma et al., 2015; Maruska et al., 2014). Since the BPG axis is critically important for reproductive competence and fertility, understanding how these GnRH1 neurons and the downstream components of the reproductive axis (e.g. pituitary, gonads) change in response to social signals has important functional implications for all vertebrates.

One candidate molecule possibly involved in both neuron changes and plasticity of the entire reproductive axis is the mechanistic target of rapamycin (mTOR; also known as mammalian target of rapamycin, target of rapamycin, FK506-binding protein 12-rapamycin-associated protein 1, FRAP1). The mechanistic target of rapamycin is a serine/ threonine protein kinase of the phosphatidylinositol 3-kinase-related protein family, and functions in many cellular processes including transcription, translation, ribosomal biogenesis, autophagy, and cell growth, proliferation, and survival (van Dam et al., 2011; Xu et al., 2016). mTOR is an evolutionarily conserved kinase found in all eukaryotes that regulates cell growth in response to various environmental and intracellular cues via formation of two distinct complexes: mTORC1 (rapamycin sensitive) and mTORC2 (rapamycin insensitive) (Laplante and Sabatini, 2009; van Dam et al., 2011; Weber and Gutmann, 2012). Several previous studies provide evidence for a role of mTOR in reproductive neuroendocrine pathways, but only in mammals (Cao et al., 2019; Morrison et al., 2007). For example, brain mTOR signaling is involved in metabolic regulation of the BPG axis by linking modulatory effects of leptin (a satiety hormone) to reproductive centers (Scanlon et al., 2003). Studies in rats show that central mTOR signaling is involved in control of puberty onset and gonadotropin secretion, and that blocking the mTOR pathway with rapamycin results in suppression of the BPG axis in both females and males (Roa et al., 2009; Roa and Tena-Sempere, 2014). Further, there is evidence for mTOR involvement at all levels of the reproductive axis in mammals, from the brain (Roa and Tena-Sempere, 2014), to gonadotropes in the pituitary (Kim et al., 2014; Roa and Tena-Sempere, 2014), to regulation of spermatogenesis and other functions in the testis (Busada et al., 2015; Jesus et al., 2017; Morrison et al., 2007; Penney and Volkoff, 2014). Thus, while mTOR is hypothesized to be a conserved regulator that controls cell growth and synaptic plasticity, its potential conserved function in regulation of the reproductive axis in non-mammalian vertebrates is unknown.

Here we analyzed the involvement of mTOR signaling in sociallyinduced plasticity of the BPG axis by exploiting the transitions in social status that occur naturally in males of the cichlid fish A. burtoni. Specifically, we hypothesized that mTOR (and indicators of its activity) would be present in the GnRH1 neurons of the brain, in the pituitary, and in the testes. We also hypothesized that transcript levels of mtor would change during male social transition from subordinate to dominant rank, and that inhibiting the mTOR pathway with rapamycin would influence all levels of the reproductive axis. Our results provide important insights into how conserved genes and signaling cascades that control cellular plasticity are translated into functional outputs in the BPG axis that regulates reproduction in all vertebrates.

\section{Materials and methods}

\subsection{Animals and collections}

Laboratory-bred adult Astatotilapia burtoni (Gunther, 1894) were maintained in aquaria under conditions that simulated their natural Lake Tanganyikan environment $\left(\mathrm{pH} 7.8-8.2\right.$, temperature $28-30^{\circ} \mathrm{C}$, conductivity 300-400 $\mu \mathrm{S} / \mathrm{cm}, 12: 12-\mathrm{h}$ light:dark cycle), and fed cichlid flakes each morning. Aquaria contained gravel-covered bottoms and halved terra cotta pots to serve as shelters and spawning territories. Fish used for staining or qPCR experiments below were netted quickly from their aquaria, measured for standard length (SL), body mass (BM), and sacrificed by rapid cervical transection. Testes were removed and weighed to calculate gonadosomatic index [GSI, = (gonad mass/body mass)*100] as a measure of reproductive investment. Brains and other relevant tissues were removed and fixed in $4 \%$ paraformaldehyde at $4{ }^{\circ} \mathrm{C}$ overnight, rinsed for several hours in $1 \mathrm{x}$ phosphate-buffered-saline (PBS), and cryoprotected in $30 \%$ sucrose made in $1 \times \mathrm{xBS}$ at $4{ }^{\circ} \mathrm{C}$ overnight. All experiments were performed in accordance with the recommendations and guidelines stated in the National Institutes of Health (NIH) Guide for the Care and Use of Laboratory Animals, 2011. The protocols were approved by the Stanford University Administrative Panel on Laboratory Animal Care (APLAC) and the Institutional Animal Care and Use Committee (IACUC) at Louisiana State University, Baton Rouge, LA.

\subsection{Tissue distribution of mtor transcripts}

To determine the distribution of mtor in different $A$. burtoni tissues, RT-PCR was performed on the following A. burtoni tissue samples collected from adult males: olfactory bulbs, pituitary, retina, saccule, gill, kidney, spleen, stomach, intestine, liver, skeletal muscle, heart, testis, and ovary (collected from females). The brain was also macro-dissected into the following parts: telencephalon, hypothalamus, remaining diencephalon and midbrain, cerebellum, hindbrain, and spinal cord as indicated in Fig. 1. Tissues were removed, rapidly frozen on dry ice, and homogenized. Total RNA was then isolated and DNase-treated using RNeasy kits (Qiagen Inc., Valencia, CA). Approximately $0.3 \mu \mathrm{g}$ total RNA from each sample was used to make cDNA with an iScript reverse transcriptase kit (Bio-Rad Laboratories, Hercules, CA). Advantage 2 Polymerase (Clontech, Mountain View, CA) was used for PCR reactions, following the Advantage 2 PCR protocol, and carried out on an iCycler thermocycler (Bio-Rad Laboratories, Hercules, CA) using the following reaction conditions: $3 \mathrm{~min}$ at $95^{\circ} \mathrm{C}$, followed by 35 cycles of $30 \mathrm{sec}$ at $95^{\circ} \mathrm{C}, 30 \mathrm{sec}$ at $60^{\circ} \mathrm{C}$, and $30 \mathrm{sec}$ at $72{ }^{\circ} \mathrm{C}$. PCR products were electrophoretically run alongside Trackit $1 \mathrm{~kb}$ DNA ladder on a $1 \%$ agarose gel containing ethidium bromide and then photographed. Negative controls included running the same procedure without any cDNA tissue template, or without reverse transcriptase, both of which produced no reaction products. The following primers were used to amplify $A$. burtoni mtor (Accension \#XM_005941648.2): forward: 5'-CACCTTAGTTC AGCAAGCTATGA-3', reverse: 5'-CTCCAGGTCTCGACACATCAGCAG-3', giving a product size of $402 \mathrm{bp}$. As a positive quality control, $A$. burtonispecific primers for the reference gene $\beta$-actin were used to amplify a 179 bp PCR product in the same samples. Representative PCR products for both mtor and $\beta$-actin were verified by sequencing (Sequetech, Mountain View, CA). 


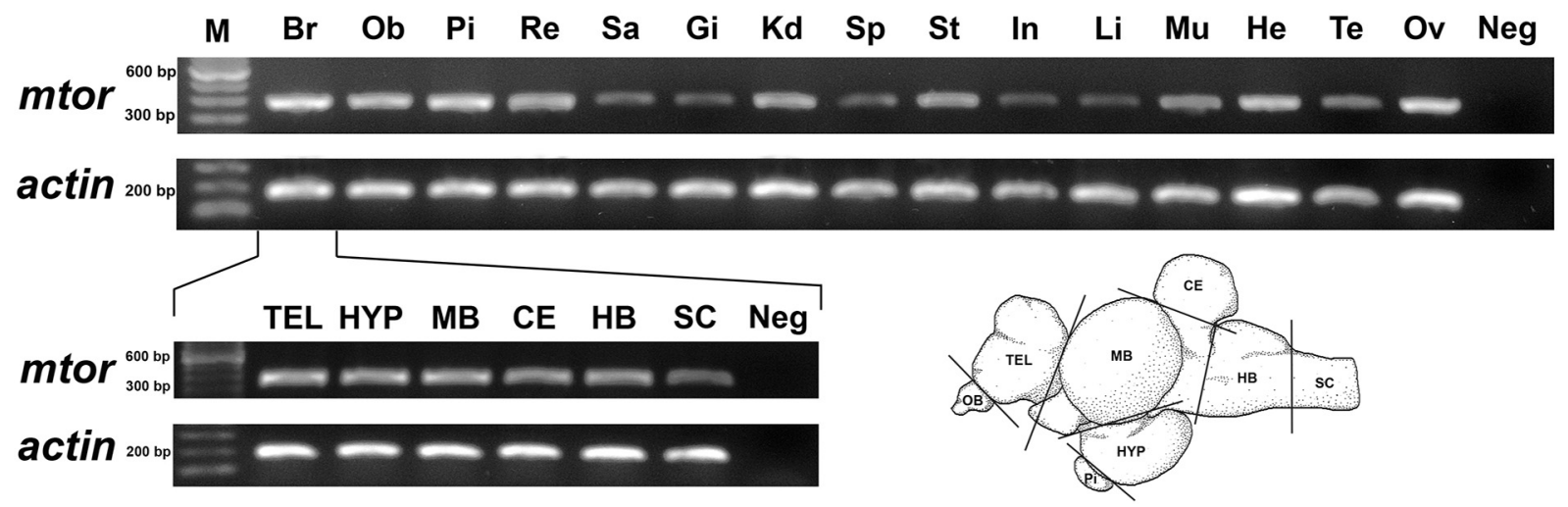

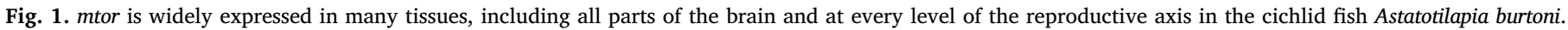

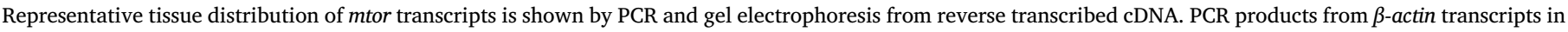

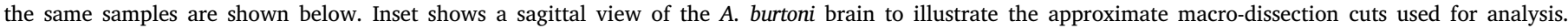

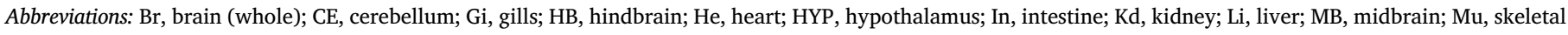

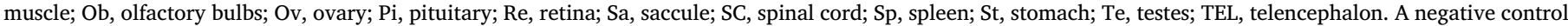
(Neg; without tissue cDNA) and DNA marker (M; 100 bp ladder) are also shown.

\subsection{Laser capture microdissection (LCM) and qPCR of single GnRH1 neurons}

To test whether individual GnRH1 neurons expressed mtor transcripts, we used single cell laser capture microdissection followed by RNA isolation, linear amplification of RNA, and reverse transcriptase (RT)-qPCR. Brains were collected and rapidly frozen, embedded in OCT media on dry ice, sectioned in the horizontal plane at $20 \mu \mathrm{m}$ on a cryostat, and collected on charged slides. Slides were then rapidly stained with cresyl violet $(75 \%$ ethanol $1 \mathrm{~min}$, water $30 \mathrm{sec}, 0.5 \%$ cresyl violet $30 \mathrm{sec}$, rinse dips in water, $75 \%$ ethanol $30 \mathrm{sec}, 100 \%$ ethanol $1 \mathrm{~min}$; all performed at $4{ }^{\circ} \mathrm{C}$ ) to facilitate identification of $\mathrm{GnRH} 1 \mathrm{neu}$ rons in the anterior preoptic area, which was based on location, size, and morphology. Only large GnRH1 neurons were selected for LCM because they are more readily and reliably identified in preoptic area (POA) sections. While GnRH1 neurons are larger in dominant compared to subordinate males, GnRH1 neurons in both social states are visibly larger than the surrounding neurons in the anterior POA and were easily identified for LCM selection. One limitation of LCM, however, is the potential for inclusion of some additional non-target cellular components, but our selection of only the largest GnRH1 cells was designed to minimize these effects. LCM was performed with a Zeiss PALM Microbeam LCM system. Neurons were marked in the PALMRobo software, and then $\sim 30 \mathrm{GnRH1}$ neurons per sample were removed with the laser microbeam and catapulted into the cap of a microcentrifuge tube containing $30 \mu \mathrm{l}$ of RNA isolation buffer that was positioned above the slide. For comparisons, we also collected $\sim 30$ non-GnRH1 neurons from the same brain sections in areas adjacent to the GnRH1 neurons, and larger areas of the preoptic area that contained $\sim 5$ to $8 \mathrm{GnRH} 1$ neurons per section in additional animals using the same protocol.

Total RNA was isolated (Arcturus Picopure RNA Isolation kit), followed by two rounds of linear amplification (Ambion MessageAmp II aRNA Amplification kit) according to kit protocols. The integrity of amplified RNA was checked on a Bioanalyzer (Agilent, RNA Pico chip), RNA was converted to cDNA (Superscript III cDNA kit, Invitrogen), and then qPCR was performed with gene-specific primers in $20 \mu \mathrm{l}$ duplicate reactions (SSoFast EvaGreen Supermix, Bio-Rad) on a Bio-Rad CFX96 machine as in our previous studies (Maruska and Fernald, 2013; Maruska et al., 2007; Moreira et al., 2019). Because linear RNA amplification can introduce a $3^{\prime}$ bias, we used mtor primers closer to the $3^{\prime}$ end of the gene for all LCM samples (forward: $5^{\prime}$-GCCACCAGTCTGTT GTATCATTGTG-3'; reverse: 5'-CTCAGTACATCGCAGTCCACTCAA-3') In addition to mtor, we also measured gnrh1 levels in all samples to verify that our collections contained (GnRH1 single cells and POA) or did not contain (non-GnRH1 cells) GnRH1 neurons. PCR Miner was used for analysis (Zuber et al., 2008), and the geometric means of the reference genes $18 \mathrm{~s}$ and glyceraldehyde 3-phosphodehydrogenase (gapdh) in the same samples were used to normalize qPCR values as in our previous studies (Maruska and Fernald, 2011; Maruska et al., 2007; Moreira et al., 2019). Throughout all above procedures, samples were kept frozen or on ice as much as possible and RNase-free solutions and glassware/plastics were used to minimize RNA degradation.

\subsection{Immunohistochemistry}

Phosphorylation of mTOR protein at Ser-2448 by the PI3 kinase/Akt signaling pathway is commonly used as a biomarker for the activation status of mTOR (Chiang and Abraham, 2005). To label GnRH1 neurons containing phosphorylated-mTOR, we developed the following protocol for double-labeling. Brains from dominant $(n=3$; GSI $=1.06 \pm 0.06)$ and subordinate $(\mathrm{n}=3$; GSI $=0.31 \pm 0.17)$ adult male $A$. burtoni were removed following rapid cervical transection, immersion fixed in $4 \%$ paraformaldehyde for $16 \mathrm{hrs}$ at $4{ }^{\circ} \mathrm{C}$, and then embedded in paraffin. Seven-micron thick sections were taken parallel to the base of the brain to optimize exposure of the GnRH1 neurons (Greenwood and Fernald, 2004) and mounted on charged slides.

Paraffin-embedded sections were placed at $60^{\circ} \mathrm{C}$ for $20 \mathrm{~min}$, incubated in xylene at room temperature for $30 \mathrm{~min}$, and then transferred sequentially into an alcohol series (100\%, 95\%, 75\%, and $50 \%$ ) for $10 \mathrm{~min}$ each at room temperature. Sections were rinsed in deionized water and phosphate buffered saline (PBS) three times. Slides were then immersed in a citrate buffer heat retrieval solution (Antigen Decloaker, pH 6.0; Biocare Medical, Concord, CA) in an electric pressure cooker (Cuisinart CPC 600, East Windsor, NJ). The slides were subjected to high pressure for $10 \mathrm{~min}$ and then briefly rinsed and stored in PBS. Nonspecific binding was blocked with a $2 \mathrm{hr}$ incubation in PBS supplemented with $0.2 \%$ bovine serum albumin, $10 \%$ normal goat serum (Invitrogen, Grand Island, NY), and 0.3\% Triton X-100. GnRH1 neurons were detected with a custom polyclonal guinea pig antibody (Thermo Scientific Pierce, Rockford, IL) generated against the $A$. burtoni mature GnRH1 peptide sequence (pyro-QHWSYGLSPG-amide). Slides were incubated with the anti-GnRH1 antibody diluted 1:1000 and antiphospho-mTOR (Ser2448) rabbit monoclonal antibody (Cell Signaling \#2976; Danvers, MA, RRID:AB_490932) diluted 1:1000 in PBS including $0.3 \%$ Triton $\mathrm{X}-100$ at $4{ }^{\circ} \mathrm{C}$. Following primary antibody incubation, slides were washed three times for $5 \mathrm{~min}$ in PBS, and incubated for $2 \mathrm{hrs}$ at room temperature with Texas Red-conjugated goat anti-guinea pig IgG (Jackson ImmunoResearch Labs., West Grove, PA) 
and DyLight 488-conjugated goat anti-rabbit IgG (Vector Labs, Burlingame, CA) diluted 1:500 in PBS including 0.3\% Triton X-100. Slides were then washed three times for $5 \mathrm{~min}$ in PBS and cover-slipped with a mounting medium containing DAPI (Vector Labs). Brain tissue stained with anti-phospho-mTOR preabsorbed with the blocking peptide used as an antigen (Cell Signaling \#1230) showed no staining. Confocal imaging was performed with a Zeiss LSM-700 confocal microscope with a z-interval of $1.0 \mu \mathrm{m}$ and the images $(2048 \times 2048$ pixels) saved as TIFF files. Maximum intensity projections of confocal zseries were made in Image $(\mathrm{NIH}$, Bethesda, MD).

\subsection{Chromogenic in situ hybridization}

To localize mtor expression in the brain, pituitary, and testis of $A$. burtoni, we used chromogenic in situ hybridization with riboprobes on cryosectioned tissue (transverse $20 \mu \mathrm{m}$ sections) following previously described protocols in A. burtoni (Butler and Maruska, 2016; Grone and Maruska, 2015; Maruska et al., 2017). Templates for riboprobes were generated by RT-PCR amplification of whole brain cDNA with genespecific primers ( $m$ tor forward: 5'-ACTAGAGGAGGTAATCCAGTA TAA-3'; mtor reverse: 5'-GGTGTCGATTCGAGCTATAAG-3'; $1000 \mathrm{bp}$ probe). Purified PCR products were then transcribed to incorporate digoxigenin (DIG)-labeled nucleotides into the sequence, followed by probe purification (GE Illustra Probe Quant G-50 microcolumns). Probes were transcribed from the T3 polymerase transcription initiation sequence that was added to the reverse (for anti-sense probes) or forward (for sense control probes) primers. Sense control probes showed no staining in the brain, pituitary, or testis.

The ISH protocol was performed as described previously in A. burtoni (Grone and Maruska, 2015; Maruska et al., 2017). Briefly, slides of cryosectioned tissue were brought to room temperature and brain sections were surrounded with a hydrophobic barrier (Immedge pen, Vector Labs). Slides were treated with the following solutions: 1xPBS ( $3 \times 5 \mathrm{~min}), 4 \%$ PFA $(20 \mathrm{~min}), 1 \times \mathrm{PBS}(2 \times 5 \mathrm{~min})$, proteinase $\mathrm{K}$ (10 min), 1xPBS (10 min), 4\% PFA (15 min), 1xPBS $(2 \times 5$ min $)$, milliQ water ( $3 \mathrm{~min}), 0.1 \mathrm{M}$ triethanolamine- $\mathrm{HCl} \mathrm{pH} 8.0$ with acetic anhydride (10 $\mathrm{min}), 1 x P B S(5 \mathrm{~min})$. This was followed by pre-hybridization (hybridization buffer without probe) for $3 \mathrm{hrs}$ in a sealed chamber at $60-65^{\circ} \mathrm{C}$, followed by replacement with probe solution (probe in hybridization buffer). Slides with DIG-labeled probe solution were covered with hybrislips and incubated overnight $(\sim 18 \mathrm{hrs})$ in a $60-65{ }^{\circ} \mathrm{C}$ oven. After hybridization, stringency washes were performed at 60-65 ${ }^{\circ} \mathrm{C}$ as follows: $2 \mathrm{x}$ saline sodium citrate (SSC): $50 \%$ formamide ( $2 \times 30 \mathrm{~min}$ ), 1:1 mixture of 2x SSC: maleate buffer with tween (MABT; $2 \times 15 \mathrm{~min})$, and MABT $(2 \times 10 \mathrm{~min})$. Slides were transferred to room temperature and washed with MABT $(2 \times 10 \mathrm{~min})$, followed by blocking of nonspecific binding with MABT containing $2 \%$ bovine serum albumin (BSA) for $3 \mathrm{hrs}$ at room temperature. After blocking, slides were incubated with anti-DIG AP antibody (Roche, RRID: AB_ 514497; diluted 1:5000 in blocking solution) overnight at $4^{\circ} \mathrm{C}$ in a sealed humidified chamber. Slides were then washed in MABT $(3 \times 30 \mathrm{~min})$, treated with alkaline phosphatase (AP) buffer $(2 \times 5 \mathrm{~min})$, and developed in NBT/BCIP solution at $37^{\circ} \mathrm{C}$ for $5 \mathrm{hrs}$. Following development, slides were treated in the following solutions: $1 x$ PBS $(3 \times 5 \mathrm{~min}), 4 \%$ PFA $(10 \mathrm{~min}), 1 \mathrm{xPBS}(3 \times 5 \mathrm{~min})$. Slides were coverslipped with aqueous mounting media (Aquamount, Lerner Labs, Pittsburgh, PA), dried flat overnight, and then edges sealed with clear nail polish. Brain sections were viewed on a Nikon Eclipse Ni microscope and photographs taken with a digital camera (Nikon DS-Fi2) controlled by Nikon Elements software. Photomicrographs were adjusted for levels, contrast, and sharpening, and any distracting artifacts were removed with the clone tool in Adobe Photoshop.

\subsection{Social ascent paradigm}

To generate males at different time points after a social opportunity that allowed them to rise in rank from subordinate to dominant status, we used an identical paradigm to our previous studies (Maruska and Fernald, 2010, 2011; Maruska et al., 2007, 2013). These fish were used to examine how mTOR expression changes in the brain, pituitary, and testes during this social transition. Briefly, subject males were socially and reproductively suppressed for 4-5 weeks in community tanks. After the suppression period, these subject subordinate males were moved to the central compartment of an experimental tank that contained one larger resident dominant male and three females. The central compartment was isolated from community tanks on either side that contained fish of mixed social status and sex. Subject males remained in the experimental tank for 2 days to maintain suppression and subordinate status. On the following morning, the resident dominant male was removed in the dark $1 \mathrm{hr}$ before light onset using infrared night vision goggles, allowing the subject subordinate male to acquire the vacant territory and rise in social rank. In this paradigm, males rising in rank intensify their body coloration and begin performing both reproductive and territorial aggressive behaviors within minutes of light onset, followed by activation of the entire BPG axis on the time course of minutes to days (Maruska, 2015; Maruska et al., 2014; Maruska and Fernald, 2018). Time of ascent was defined as the time between light onset and when subject males produced dominance behaviors at a rate of three behaviors per minute. Subject fish were then sacrificed at 0.5, 6, 24, 72, and $120 \mathrm{hrs}$ after social ascent. Stable subordinate and stable dominant males were also sampled as comparisons to ascending fish, and were generated and collected as previously described (Maruska and Fernald, 2010).

\subsection{Quantitative PCR ( $q P C R$ ) during social ascent}

To examine changes in mtor expression in the pituitary and testis, we performed $\mathrm{qPCR}$ in stable subordinate, stable dominant, and ascending male individuals sampled at various time points following a rise in social rank. Since these were the same animals used in our previous qPCR studies that examined other gene expression changes during social ascent in the pituitary and testes, the methods for tissue collection, qPCR, and analyses using PCR Miner (Zuber et al., 2008) were identical and are described in detail in (Maruska and Fernald, 2011; Maruska et al., 2007). Primers for mtor were commercially synthesized: forward 5'-ACCAGCCTATCATTCGCATCCAGT-3'; reverse 5'-TCAACAGGAACATGAACTCGTGGC-3' (120 bp product). The geometric mean of the reference genes $18 \mathrm{~s}$ and gapdh were used to normalize mtor expression, as done previously (Maruska et al., 2012; Maruska and Fernald, 2011; Maruska et al., 2007).

\subsection{Intraperitoneal (IP) rapamycin injections}

To examine the effects of inhibiting the mTOR pathway at the levels of the brain, pituitary, and testes, we used intraperitoneal injections of rapamycin in dominant males. IP injections were used to mimic the systemic delivery of rapamycin used in clinical settings to examine whether similar effects on reproductive function were observed in the fish. Fish were anesthetized in ice cold-cichlid system water, weighed for body mass, intraperitoneally injected with 8-10 $\mu \mathrm{l}$ of either $5 \mu \mathrm{g} / \mathrm{g}$ BM rapamycin (LC Labs, Woburn, MA; \#R-5000; stock dissolved in DMSO and final concentration made in saline) or vehicle (DMSO and saline), and placed in warmed cichlid-system water for recovery. To test for reproductive axis dysfunction, we measured the following: p-p70s6 kinase protein levels in the whole brain and testes via western blot (see below), and circulating LH and FSH levels as a measure of pituitary function via ELISA. The small size of the pituitary did not allow for reliable western blot detection of p-p70S6 kinase protein levels, so we used plasma LH and FSH levels to measure pituitary function. Methods for LH and FSH ELISAs were identical to those used in our previous study and details can be found in (Maruska et al., 2007). Fish were sacrificed at $48 \mathrm{hrs}$ after rapamycin $(\mathrm{SL}=51.5 \pm 1.04 \mathrm{~mm}$, 
$3.61 \pm 0.26 \mathrm{~g}, \quad \mathrm{GSI}=0.90 \pm 0.15) \quad$ and $\quad$ vehicle $\quad(\mathrm{SL}=52.8 \pm$ $2.49 \mathrm{~mm}, \mathrm{BM}=4.11 \pm 0.37 \mathrm{~g}, \mathrm{GSI}=0.94 \pm 0.28$ ) injections, and tissues (brain, testes, serum) were removed and frozen at $-80{ }^{\circ} \mathrm{C}$ prior to analysis.

\subsection{Western blots}

To determine whether the mTOR inhibitor rapamycin disrupted activity of the mTOR pathway in the reproductive axis of $A$. burtoni, we used western blotting to measure levels of phosphorylated p70S6 kinase in whole brains and testes. Whole brains were removed and rapidly frozen on dry ice. Protease (Mini Protease Inhibitor Cocktail; Roche) and phosphatase (PhosSTOP; Roche) inhibitors were added to the lysis buffer ( $2 \%$ SDS in $1 \mathrm{x}$ TBS) for protein isolation, brains were homogenized, and then centrifuged for $90 \mathrm{~min}$ at $13,000 \mathrm{rpm}$ at $4{ }^{\circ} \mathrm{C}$. Supernatant was removed, protein concentration measured with a Qubit protein assay system (Thermo Fisher, Inc.), $40 \mu \mathrm{g}$ of protein per sample was run on a $10 \%$ Bis-Tris Precast Gel with MOPS running buffer (Bio-Rad, Inc.), and then transferred to a $0.45 \mu \mathrm{m}$ nitrocellulose membrane filter (Invitrogen \#LC2001). Coomassie blue staining was used to verify protein loading across samples. Membranes were blocked overnight at $4{ }^{\circ} \mathrm{C}$ on a rotator (1x TBS, $0.1 \%$ Tween-20, $5 \%$ BSA), and incubated with primary antibody (1:1000 made in blocking solution) for $1.5 \mathrm{hrs}$ at room temperature on a rotator. The p-p70S6 kinase primary antibody (Cell Signaling Technologies, Inc. \#9205; rabbit polyclonal made by immunizing animals with synthetic phosphopeptide corresponding to residues around Thr389 of human p70S6 kinase; RRID: AB_330944) detects endogenous levels of p70S6 kinase only when phosphorylated at Thr389 (antibody also detects p85S6 kinase when phosphorylated at the analogous site Thr412). Following primary antibody, blots were washed (1x TBS with $0.1 \%$ Tween- 20 on rotator at room temperature, $3 \times 15 \mathrm{~min})$, incubated in secondary antibody (1:1000 made in blocking solution, Goat HRP anti-rabbit, \#PI-1000, Vector Labs) for $1 \mathrm{hr}$ at room temperature on a rotator, washed (1x TBS with $0.1 \%$ Tween-20, 5x15min), and developed with ECL Western Blotting Detection kit (Amersham). Blots were then imaged by exposure on Kodak BioMax XAR film in a darkroom, and developed by an automatic developing machine. To quantify the relative intensity of p-p70S6 kinase bands from western blots, we scanned developed films and used the Gel Analysis method in ImageJ outlined in the following protocol tutorial: http://lukemiller.org/index.php/2010/11/analyzing-gels-andwestern-blots-with-image-j/. All blots contained multiple representative samples from both control vehicle and rapamycin-injected fish.

\subsection{Intracerebroventricular (ICV) rapamycin injections}

To test whether inhibition of the mTOR pathway would affect the typical socially-induced hypertrophy of GnRH1 neurons during a rise in social rank, we used intracerebroventricular (ICV) injections of rapamycin in subordinate males prior to social ascent. Fish were selected and treated as above in the ascension paradigm. Resident dominant males were then removed from the experimental tank $1 \mathrm{hr}$ prior to lights on, providing an opportunity for the subject subordinate male to acquire the territory and rise in social rank (Maruska and Fernald, 2010). The subject subordinate males were then removed $\sim 15 \mathrm{~min}$ prior to lights on, anesthetized in ice-cold cichlid system water, and injected directly into the 3rd ventricle with $2 \mu \mathrm{l}$ of rapamycin $(25 \mathrm{mg}$ / $\mathrm{ml}$ dissolved in DMSO; fish $\mathrm{SL}=62.8 \pm 2.4 \mathrm{~mm}, \mathrm{BM}=6.06 \pm$ $0.61 \mathrm{~g}, \mathrm{GSI}=0.61 \pm 0.17, \mathrm{n}=5$ ) or vehicle control (DMSO; fish $\mathrm{SL}=60.3 \pm 4.2 \mathrm{~mm}, \mathrm{BM}=5.72 \pm 1.2 \mathrm{~g}, \mathrm{GSI}=0.44 \pm 0.07, \mathrm{n}=4)$ with a guided 30-gauge Hamilton syringe inserted through the dorsal braincase. In pilot experiments, we validated the stereotactic injection location using Evans blue dye ( $0.1 \%$ in $0.9 \%$ saline), sectioned the brain in the transverse plane at $300 \mu \mathrm{m}$ on a cryostat, and verified presence of stain within the 3 rd ventricle. Following injection, subject subordinate males were allowed to recover for $\sim 15 \mathrm{~min}$ and then placed back in the experimental tank where they took over the territory and ascended in social rank after the lights turned on. Fish remained in the experimental tank where they behaved as dominant males and were sacrificed at 3 days after social ascent. Three days was chosen because GnRH1 neurons have increased in size by this time point (Maruska et al., 2014). Territorial and reproductive behaviors were also quantified prior to sacrifice as described previously (Maruska and Fernald, 2010) and showed no differences between control and rapamycin-injected ascending males (territorial behaviors: $t$-test, $\mathrm{df}=7, \mathrm{t}=0.271$, $\mathrm{p}=0.794$; reproductive behaviors: $\mathrm{MW}$ rank sum test, $\mathrm{df}=7$, $\mathrm{U}=5.50, \mathrm{p}=0.271$ ).

To test whether ICV rapamycin treatment influenced GnRH1 neuron size during social ascent, we cryosectioned brains in the transverse plane at $20 \mu \mathrm{m}$, collected sections on alternate charged slides, and stained one set of slides to label GNRH-containing cells (rabbit polyclonal anti-LHRH/GnRH \#20075, ImmunoStar Inc. RRID: AB_572248, at 1:1000; produced by immunization with synthetic LHRH coupled to keyhole limpet hemocyanin with carbodiimide linker) using standard immunohistochemistry protocols described previously (Maruska, 2009; Maruska and Tricas, 2011; Maruska et al., 2012). As a negative control, $\mathrm{GnRH}$ antibody was preabsorbed with immunizing peptide and also applied to brain tissue, which eliminated all reaction product in the preoptic area where GnRH1 neurons are located. This antibody also shows identical labeling of GnRH1 neurons to that observed with in situ hybridization, other GnRH antibodies, and GnRH1:eGFP transgenic fish shown in previous studies of $A$. burtoni (Bryant et al., 2016; Butler and Maruska, 2019; Davis and Fernald, 1990; Francis et al., 1993; Ma et al., 2015). We then measured cross-sectional areas of all GnRH-immunoreactive somata in the preoptic area that showed a distinguishable perimeter and visible nucleus (ImageJ).

\subsection{Statistical analyses}

Comparisons among groups were performed with 1-way ANOVA ( $>2$ groups) or Student's t-tests ( 2 groups). In some cases, data were $\log$ transformed prior to analysis to meet the parametric assumptions of normality and equal variance. When data did not meet these assumptions even after transformation, non-parametric tests were used (MannWhitney test, MW). Pearson correlation tests were also used to test for relationships between variables. Statistical analyses were performed with SigmaPlot 12.3 (Systat, Inc., San Jose, CA).

\section{Results}

3.1. Mtor transcripts are widely expressed throughout the brain and other tissues of $A$. burtoni

We used RT-PCR to localize mtor transcripts in different tissues from adult male $A$. burtoni (Fig. 1). Transcripts for mtor were detected in all tissues examined, including the retina, saccule (auditory endorgan), gill, kidney, spleen, stomach, intestine, liver, skeletal muscle, heart, testis, and ovary (from adult female). All macro-dissected regions of the brain (olfactory bulbs, pituitary, telencephalon, hypothalamus, diencephalon and midbrain, cerebellum, hindbrain, and spinal cord) also contained mtor mRNA expression.

\section{2. mTOR plays a role in GnRH1 neuron plasticity during social transition in male A. burtoni}

We used laser capture microdissection (LCM) followed by qPCR to measure mtor expression in GnRH1 neurons of the preoptic area (POA). mRNA levels of mtor were highest in isolated GnRH1 neurons, followed by sections of the entire POA (which also contained GnRH1 cells), with negligible levels detected in non-GnRH1 cells sampled from adjacent regions of the same brain sections (1 way ANOVA, $F=78.026$, 

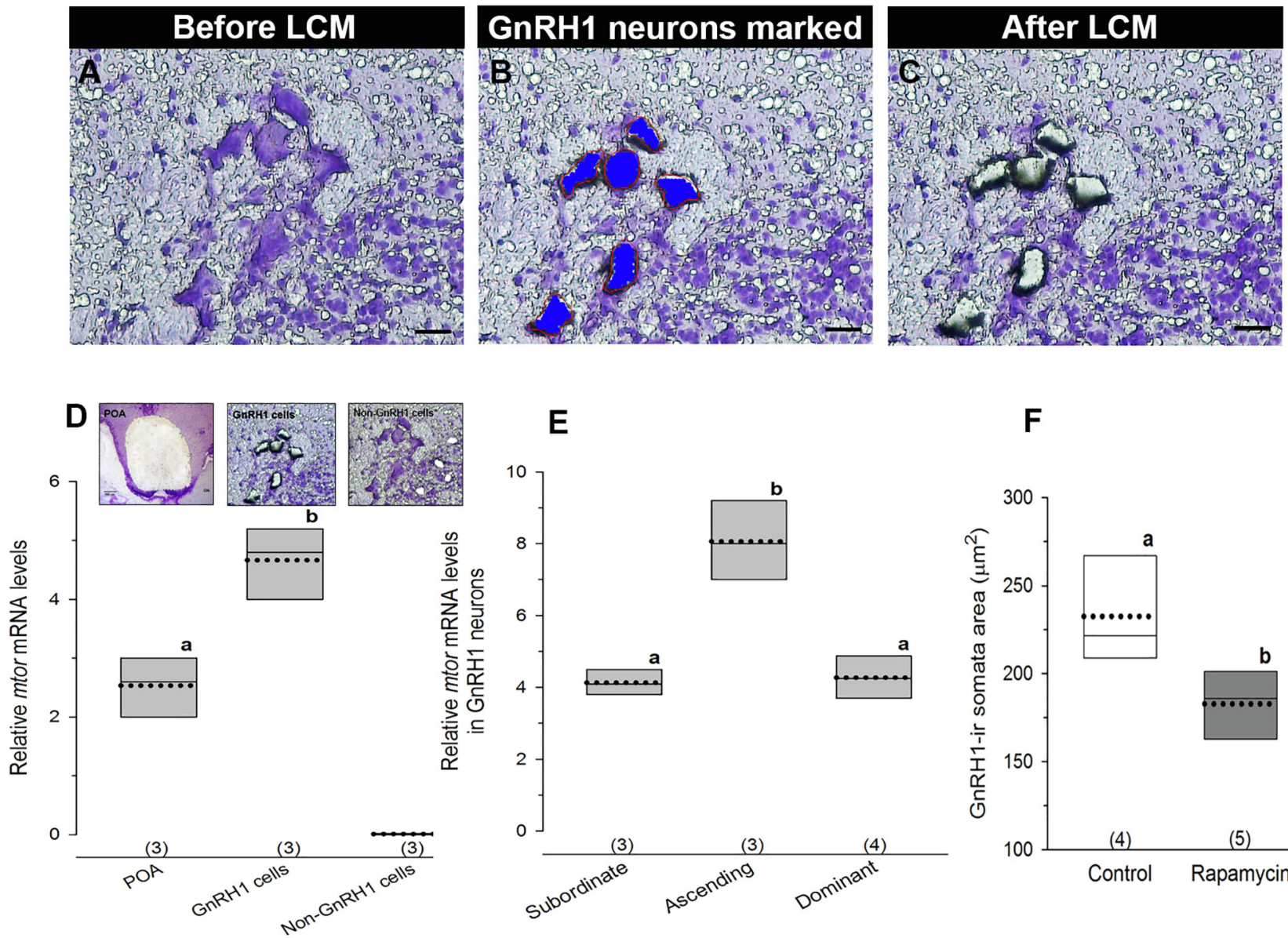

E

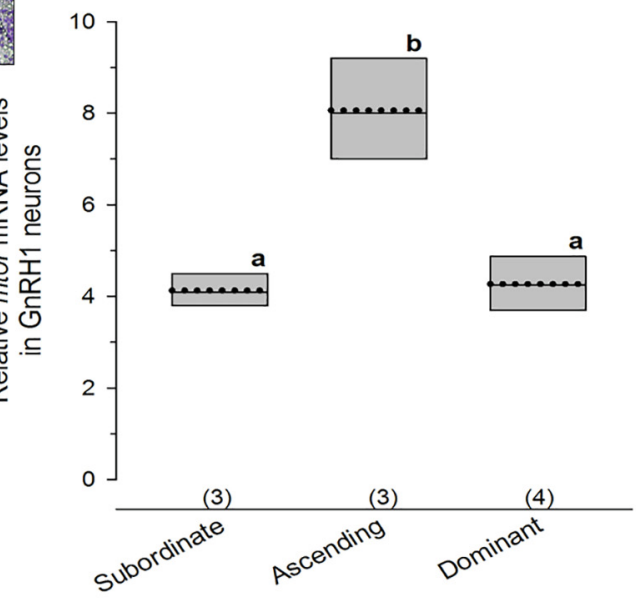

$\mathbf{F}$

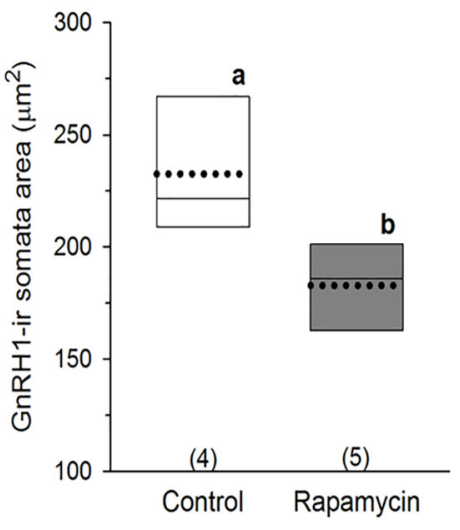

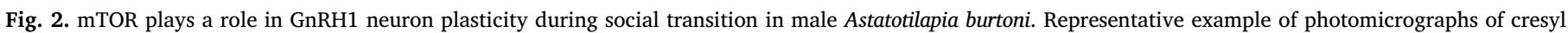

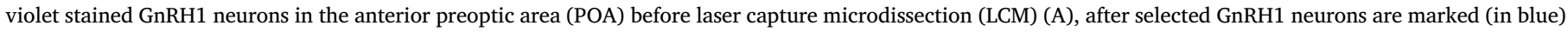

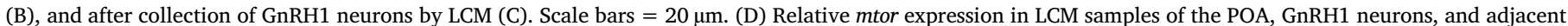

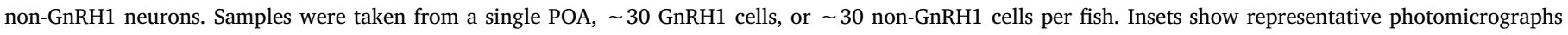

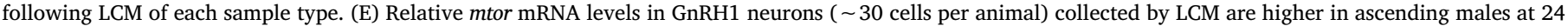

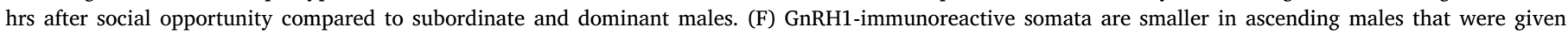

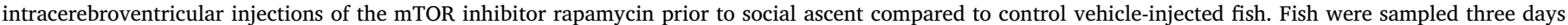

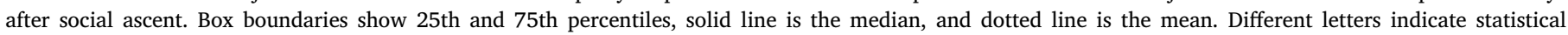

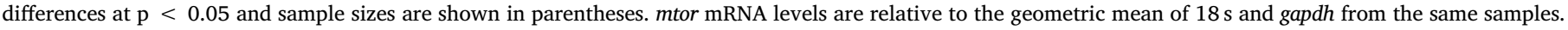

$\mathrm{df}=2,6, \mathrm{p}<0.001 ;$ SNK test $\mathrm{p}<0.001)($ Fig. 2A-D). We compared mtor levels in LCM-isolated single GnRH1 neurons of subordinate, dominant, and ascending males collected at $24 \mathrm{hrs}$ after social ascent, a time when increased gnrh1 mRNA production and somata size can already be detected (Maruska et al., 2014). Ascending males that rose in social rank had higher mtor mRNA levels in captured GnRH1 neurons compared to both subordinate and dominant stable states (1-way ANOVA, $\mathrm{F}=27.502, \mathrm{df}=2,7, \mathrm{p}<0.001$, SNK test $\mathrm{p}<0.001$ ) (Fig. 2E).

To test whether disruption of mTOR activity would change the functional activity of the A. burtoni BPG axis, we used the mTOR pathway inhibitor rapamycin. We intraperitoneally (IP)-injected rapamycin, which is a macrolide compound produced by the bacterium Streptomyces hygroscopicus and is a well-known inhibitor of the mTORC1 pathway (Fig. 3A). Rapamycin acts by associating with FKBP12 (12 kDa FK506-binding protein), which then inhibits the mTORC1 complex, leading to decreased activity of P70S6 kinase. P70S6 kinase lies in a mitogen-activated signaling pathway that is downstream of mTOR, and phosphorylation at threonine 389 (Thr389, the linker domain) is critical for kinase function to phosphorylate the S6 protein of the 40S ribosomal subunit involved in translational control of some mRNAs involved in cell growth (Roa et al., 2010) (Fig. 3A). Thus, measurement of phosphorylated p70S6 kinase is commonly used as an indicator of
mTOR signaling activity. Fig. 3B shows an example of p-p70S6 kinase western blot analysis of whole brains from a control vehicle treated male and males sampled at 24-48 $\mathrm{h}$ after rapamycin treatment. Levels of the $70 \mathrm{kDa}$ p-p70S6 kinase are decreased after rapamycin treatment, indicating reduced mTOR pathway activity. We chose to sample the tissue at $48 \mathrm{hrs}$ post-injection because previous studies identified this time point as when most relevant physiological changes occur in the brain, pituitary, bloodstream, and testes of this species (Maruska, 2014; Maruska, 2015; Maruska et al., 2014; Maruska and Fernald, 2018).

Could inhibition of the mTOR signaling pathway with rapamycin alter the typical GnRH1 somata hypertrophy observed after social ascent in A. burtoni? To test this, we used intracerebroventricular (ICV) injections of rapamycin or control vehicle solutions into the third ventricle of subordinate males, and then provided a social opportunity for them to rise in rank to dominant reproductive status. Rapamycintreated ascending males had smaller GnRH1 somata compared to vehicle control males at 3 days after social ascent (Student's $t$-test, $\mathrm{t}=2.813, \mathrm{df}=7, \mathrm{p}=0.026)(\mathrm{Fig} .2 \mathrm{~F})$, demonstrating reduction of the canonical neuron hypertrophy when mTOR was inhibited. 

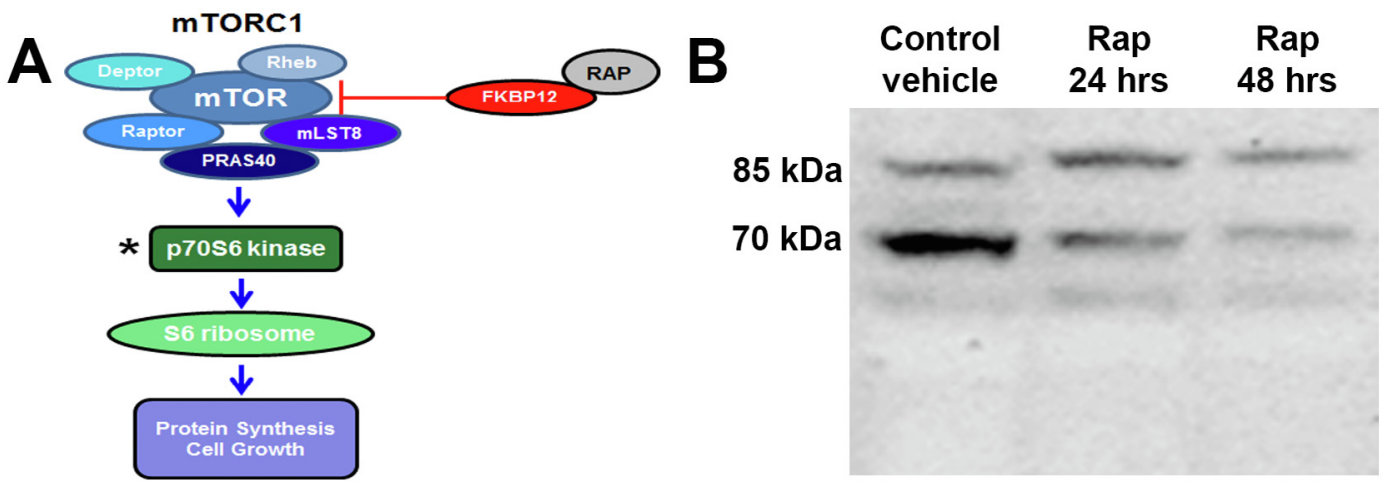

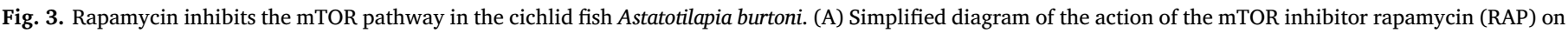

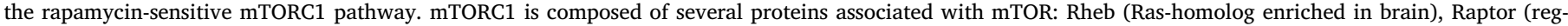

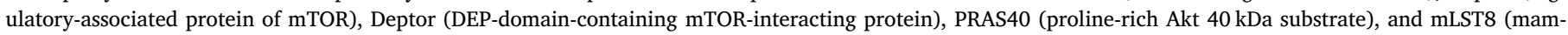

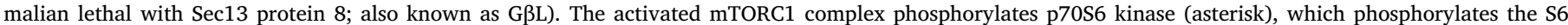

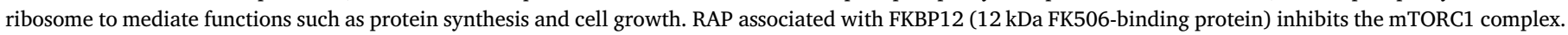

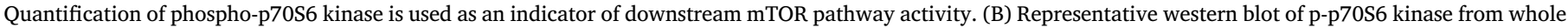

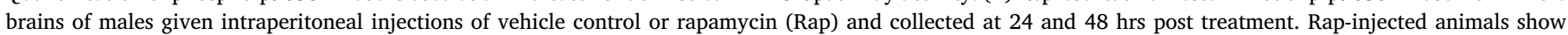
reduced expression of p-p70S6 kinase. Bands for the p-p70S6 and p-p85S6 isoforms detected by the antibody are shown.

\subsection{Phosphorylated mTOR occurs in GnRH1 neurons in dominant but not subordinate males}

To ask whether phosphorylation of mTOR protein (a proxy of activity) in GnRH1 neurons differed with social status, we used doublelabel immunohistochemistry for phospho-mTOR and GnRH1. GnRH1 neurons in dominant males showed cytoplasmic phospho-mTOR label, while label was essentially absent from GnRH1 neurons in subordinate males (Fig. 4). This indicates higher activity of the mTOR pathway in dominant individuals, and minimal to no activity in reproductivelysuppressed subordinate males.

3.4. mTOR transcripts are localized to specific regions of the pituitary and testis in A. burtoni

For mtor to act at downstream sites of the reproductive axis, it must be expressed in the pituitary and testes. We used chromogenic in situ hybridization to localize mtor transcripts in these tissues (Fig. 5). In the testes, mtor was expressed in germ cells of most spermatogenic stages (spermatogonia, spermatocytes, early spermatids) that reside inside membrane-bound spermatocysts of the fish testis (Fig. 5A-D). However, expression of mtor appeared absent in late-stage spermatids and the mature sperm located in the center of testicular tubules, and was scarce or absent from the interstitial tissue found between spermatocysts where Leydig cells reside (Fig. 5C). We were unable to determine whether mtor was present in Sertoli cells, as found in mammals (Jesus et al., 2016). In the pituitary, mtor mRNA was detected throughout the proximal pars distalis (PPD), which contains the FSH- and LH-producing cells, but was essentially absent from the pars nervosa (Fig. 5E). Some concentration of mtor label was noticeable at the boundary between the pars nervosa (PN) and the PPD, a region shown in a previous A. burtoni study to express $\mathrm{GnRH}$ receptors and growth hormone-
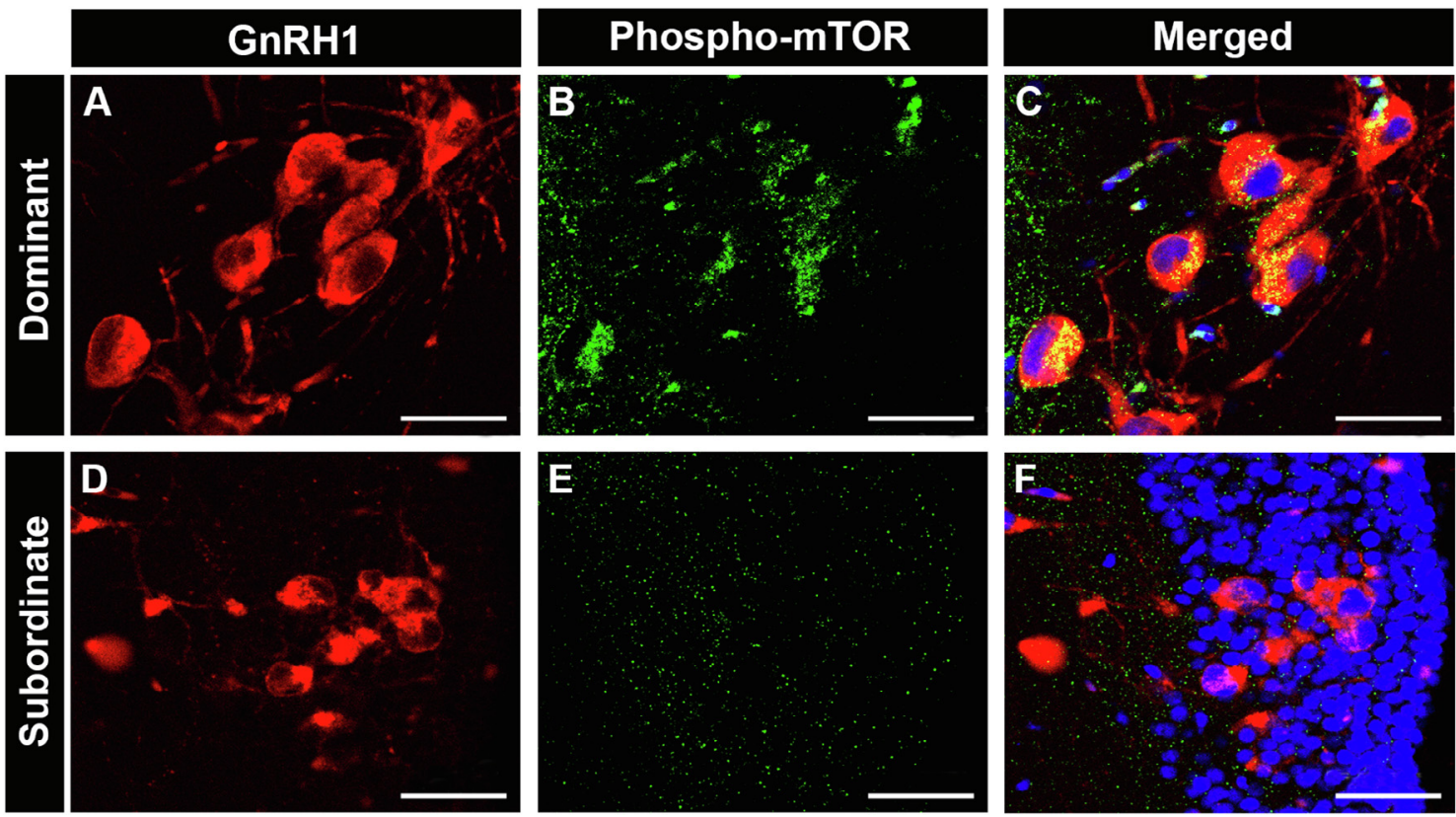

Fig. 4. Phosphorylated mTOR is co-localized in GnRH1 neurons of dominant but not subordinate male Astatotilapia burtoni. Confocal images in the anterior preoptic area of dominant (A-C) and subordinate (D-F) males show labeling with antibodies for GnRH1 (A, D), phospho-mTOR (B, E), and merged images counterstained with DAPI (C, F). Scale bars $=28 \mu \mathrm{m}$. 

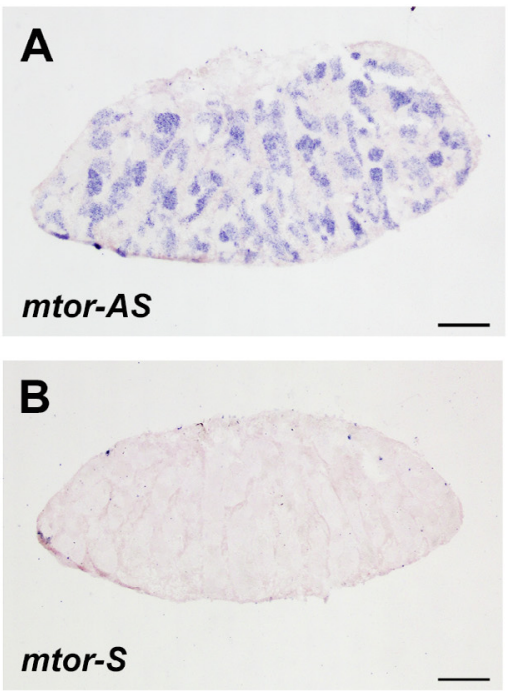
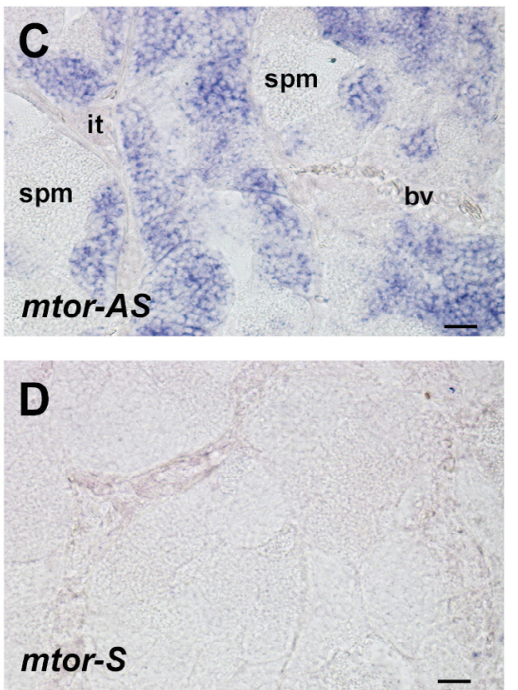
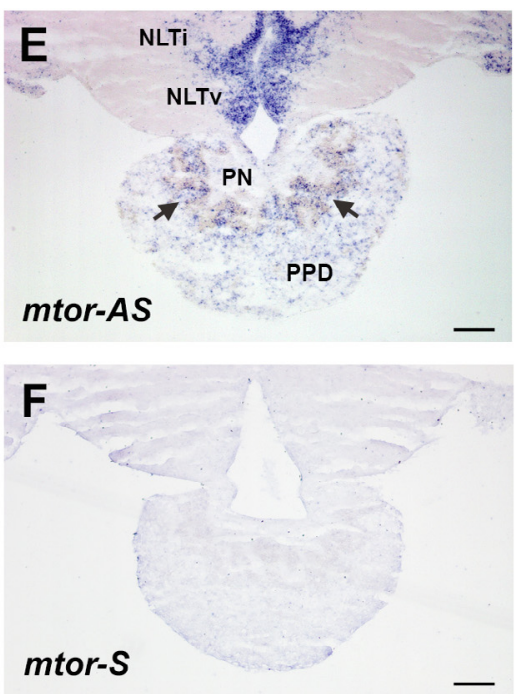

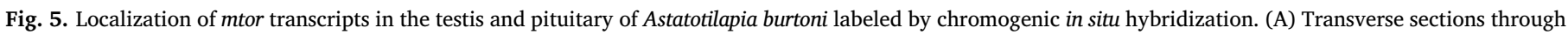

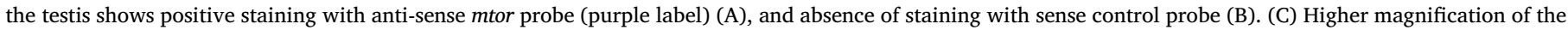

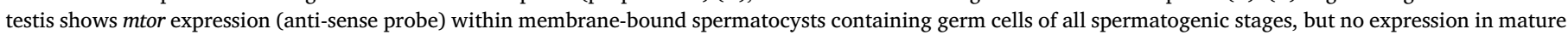

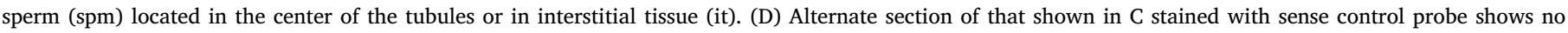

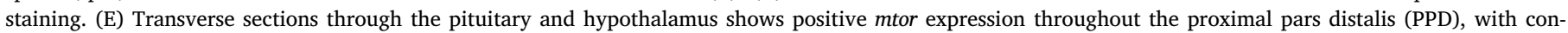

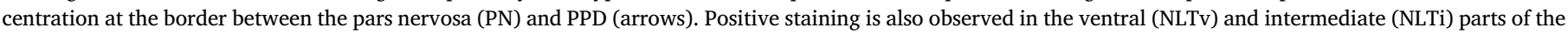

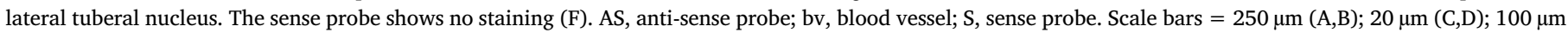
(E,F).

producing cells (Flanagan et al., 2007).

\subsection{Expression of mtor in the pituitary and testes changes during social} ascent in male A. burtoni

To determine whether mtor expression in the pituitary and testes differed as a function of social status, we used qPCR to measure mtor mRNA levels in subordinate, dominant, and ascending males collected at different time points after rising in social rank (Figs. 6 and 7). In the pituitary, levels of mtor were higher at 72-120 hrs after social ascent compared to all other time points, and compared to stable dominant and stable subordinate males (1-way ANOVA, $\mathrm{F}=6.045$, $\mathrm{df}=6,70$, $\mathrm{p}<0.001$; SNK test $\mathrm{p}<0.01$ ) (Fig. 6A). Relative mRNA levels of mtor in the pituitary were also positively correlated with mRNA levels of luteinizing hormone $(\operatorname{lh} \beta)$ across all time points (Pearson correlation, $r=0.78, \mathrm{p}=0.039)$, but not follicle stimulating hormone $(f \operatorname{sh} \beta)$ (Pearson correlation, $r=0.63, \mathrm{p}=0.132$ ) (Fig. 6B). In the testes, mtor levels were higher at $120 \mathrm{hrs}$ after ascent compared to all other time points, and compared to stable dominant and stable subordinate males (1-way ANOVA, $\mathrm{F}=9.386, \mathrm{df}=6,67, \mathrm{p}<0.001 ; \mathrm{SNK}<0.01$ ) (Fig. 7A). Further, mtor levels in the testes were positively correlated with mRNA levels of FSH receptor ( $f s h r$ ) across all time points (Pearson correlation, $r=0.97, \mathrm{p}<0.001$ ) (Fig. 7B), but not LH receptor (lhr) (Pearson correlation, $r=0.65, \mathrm{p}=0.113$ ).

\section{6. mTOR inhibition impacts all levels of the BPG axis in male A. burtoni}

To test whether inhibition of the mTOR pathway would impact the BPG axis at multiple levels, we used IP injections of the mTOR inhibitor rapamycin and measured indicators of brain, pituitary, and testes
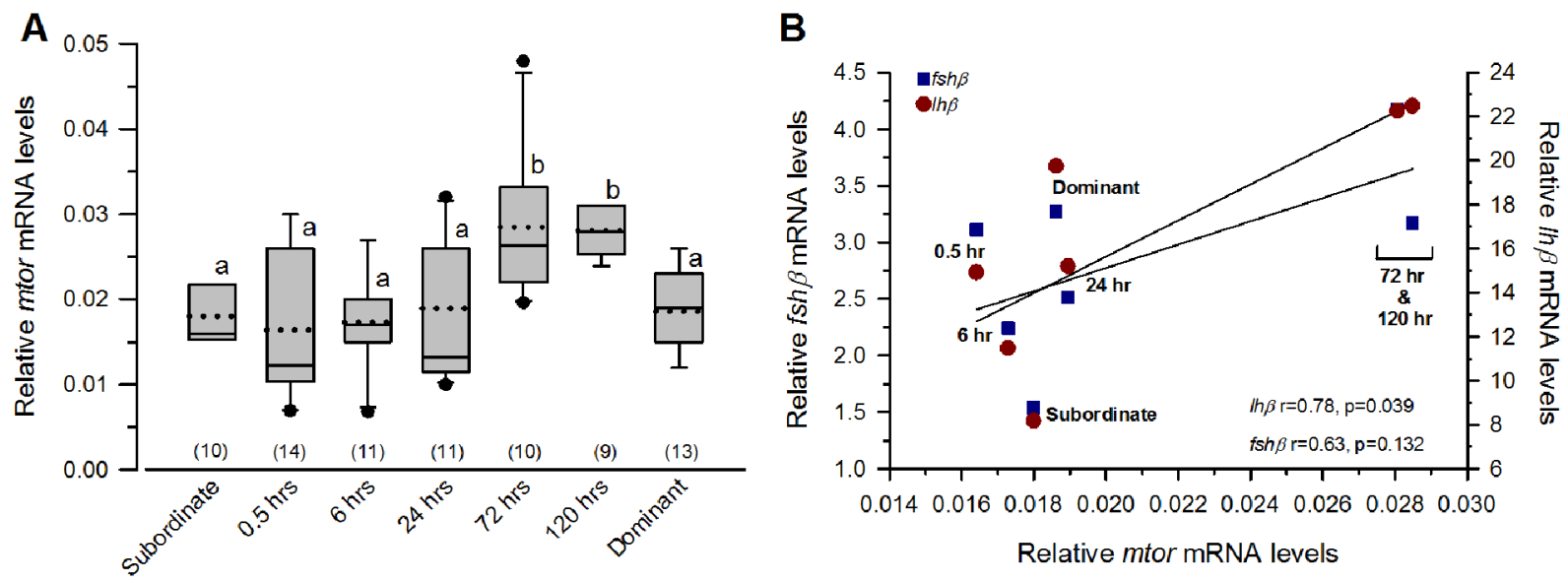

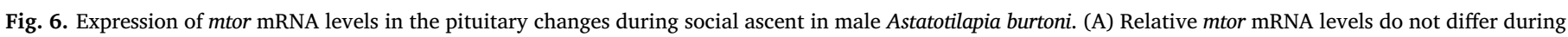

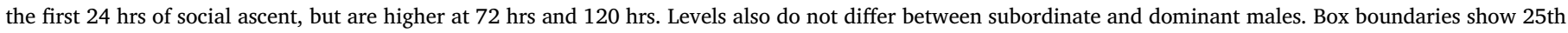

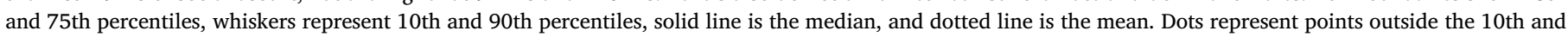

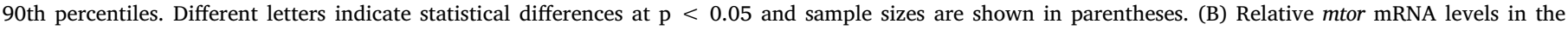

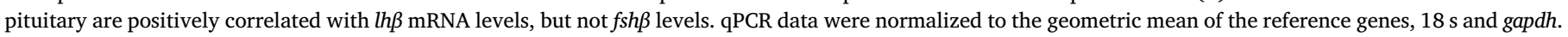



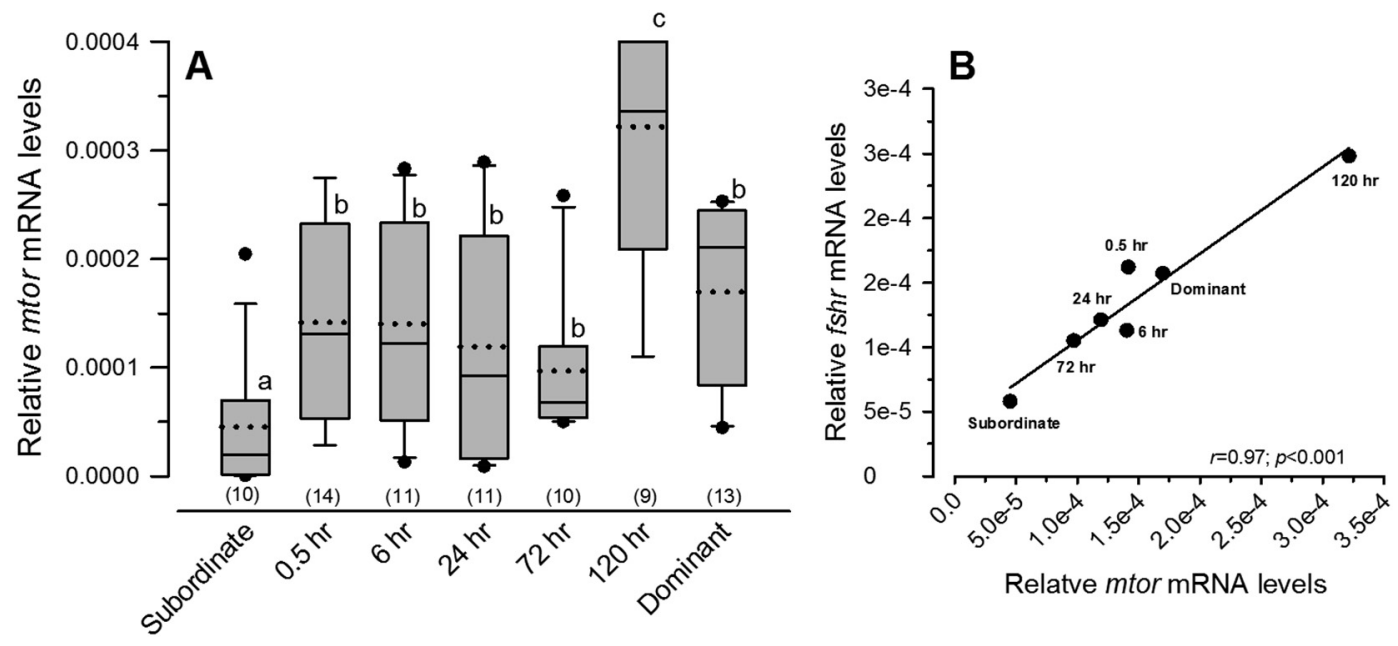

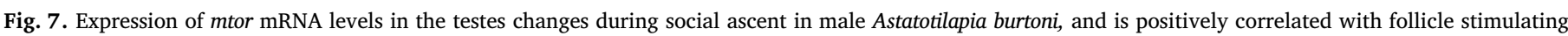

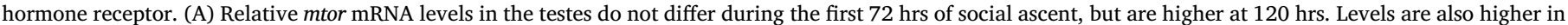

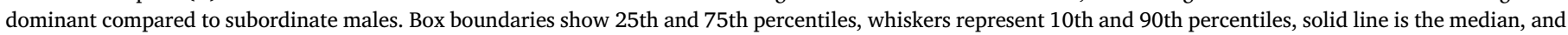

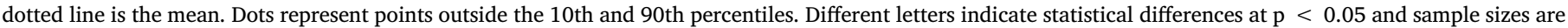

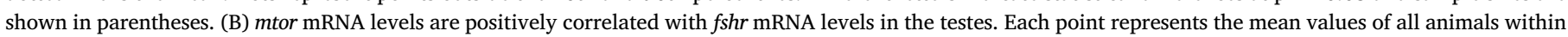
that social time point. qPCR data were normalized to the geometric mean of the reference genes, $18 \mathrm{~s}$ and gapdh.

mTOR activity (Fig. 8). In whole brains, levels of p-p70S6 kinase, a downstream indicator of mTOR pathway activity, were lower in rapamycin-treated males than in vehicle-treated males (Student's $t$-test, $\mathrm{t}=7.889, \mathrm{df}=8, \mathrm{p}<0.001$ ) (Fig. 8A). Similarly, levels of p-p70S6 kinase in the testes were also lower in rapamycin-treated males compared to control vehicle-treated males (Student's $t$-test, $t=3.802$, $\mathrm{df}=10, \mathrm{p}=0.0017$ ) (Fig. 8B). To test for functional rapamycin effects in neuroendocrine signaling, we also measured circulating levels of $\mathrm{LH}$ and FSH. Plasma levels of LH (Student's $t$-test, $\mathrm{t}=-3.002$, $\mathrm{df}=8$, $\mathrm{p}=0.017$ ) and FSH (Student's $t$-test, $\mathrm{t}=-4.355, \mathrm{df}=13, \mathrm{p}<0.001$ ) were higher in rapamycin-treated males compared to vehicle-treated males (Fig. 8C-D), possibly due to dysregulation of feedback mechanisms to the pituitary. Thus, inhibition of the mTOR pathway via rapamycin had measureable effects at all levels of the A. burtoni BPG axis.

\section{Discussion}

We show that the mTOR-signaling pathway is involved in sociallyinduced plasticity of the BPG axis in a social non-mammalian vertebrate. These are the first non-mammalian data to implicate a role for the mTOR pathway in regulation of multiple levels of the male reproductive axis. We also demonstrate that the mTOR pathway is involved in the socially-induced GnRH1 neuron hypertrophy in A. burtoni. Collectively, these results provide support for the highly conserved function of mTOR signaling in the function and plasticity of the neuroendocrine reproductive axis from fishes to mammals.

Transcripts for mTOR were ubiquitous throughout the brain and peripheral tissues of $A$. burtoni, including expression at multiple levels of the reproductive axis (pituitary, testes). While other studies in fishes confirm widespread tissue distribution, and have examined some aspects of mTOR function (e.g. development, epithelial morphogenesis, regulation of food intake, cardiomyopathy, fin regeneration) (Ding et al., 2011; Hirose et al., 2014; Jiang et al., 2013; Makky and Mayer, 2007; Makky et al., 2007; Pullen and Thomas, 1997), no previous studies have focused on its role in reproduction. Here we provide evidence, by multiple techniques (RT-PCR, qPCR, LCM-qPCR, IHC, ISH, western blot), that the mTOR-signaling pathway is present in GnRH1 neurons, in the pituitary, and in the testes of $A$. burtoni. Recent interpretations of mTOR function propose that MTOR regulation should be viewed as a reflection of cell type- and basal state-dependent conditions (Xu et al., 2016). Thus while our results highlight the potential for
mTOR-mediated regulation at every level of the reproductive axis, exact functions and mechanisms in diverse tissues or cells may differ and their cell-specific role(s) should be the focus of future study.

We detected mtor transcripts in LCM-isolated GnRH1 neurons, and show that mtor mRNA levels are higher in ascending males $24 \mathrm{hrs}$ after receiving an opportunity to rise in rank to dominant status. To our knowledge, this is the first study to implicate mTOR signaling in GnRH1 neurons. There is evidence, however, for mTOR-dependent signaling mechanisms in immortalized hypothalamic GnRH cells (GT1-7), where mTOR was shown to act as a sensor of amino acids (Mukherjee et al., 2015). Further, studies in mammals implicate mTOR as a central regulator of puberty onset via modulation of the hypothalamic kisspeptin1 system, which acts upstream of GnRH1 signaling (Roa and TenaSempere, 2014). When subordinate male A. burtoni rise in rank, the socially-induced transition is typified by GnRH1 neuron cell hypertrophy, increased dendritic arbor complexity, and cell interconnections via electrotonic conduction (Davis and Fernald, 1990; Ma et al., 2015; Schell et al., 2016), possibly to accommodate increased inputs from upstream signaling pathways, as well as up-regulated production of GnRH1 and other proteins. Thus, the elevated mtor mRNA levels observed during social ascent may be needed to increase both mTORC1 and mTORC2 complexes to accommodate the morphological and metabolic changes that occur in these GnRH1 neurons during this period, which then facilitates the rapid up-regulation of male reproductive physiology. For example, mTORC1 is involved in protein translation and linking metabolic signals to cellular function (Laplante and Sabatini, 2009; Xu et al., 2016), mTORC2 can regulate the organization of the actin cytoskeleton to control cell growth (Jacinto et al., 2004), and both complexes are involved in dendritogenesis (Sosnowski et al., 2000). These functions all likely occur within these GnRH1 neurons during $A$. burtoni social transitions, and may in part be mediated by these mTOR complexes. The fact that we observed phosphorylatedmTOR protein in GnRH1 neurons of dominant males, but not subordinate males, suggests that these mTOR-signaling cascades within GnRH1 neurons are also necessary to maintain the more active reproductive axis associated with dominant status.

We also show that inhibition of the mTORC1 pathway with ICV injection of rapamycin prior to social ascent results in smaller GnRH1 somata at a time point that typically shows increased GnRH1 somata size and increased gnrh1 mRNA production (Maruska et al., 2014). This suggests that mTOR-signaling pathways are involved in the socially- 
A

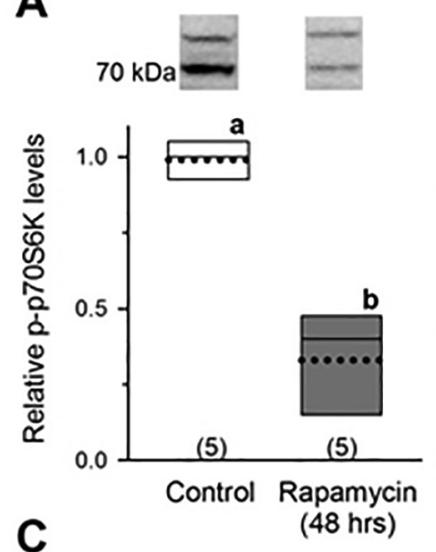

B
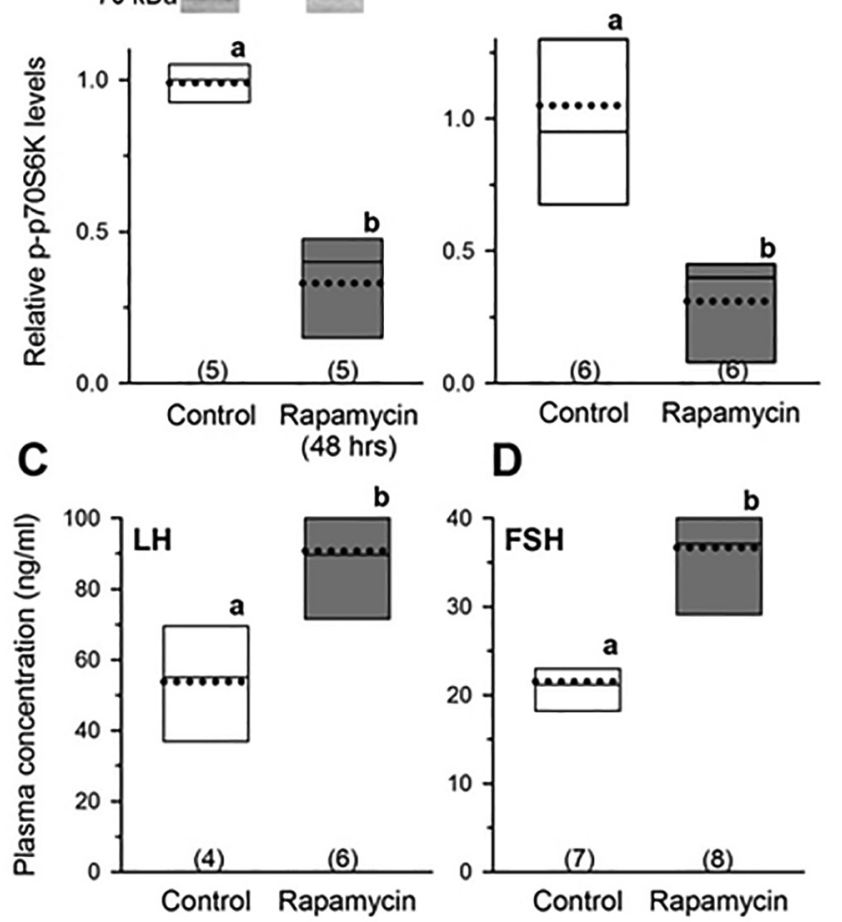

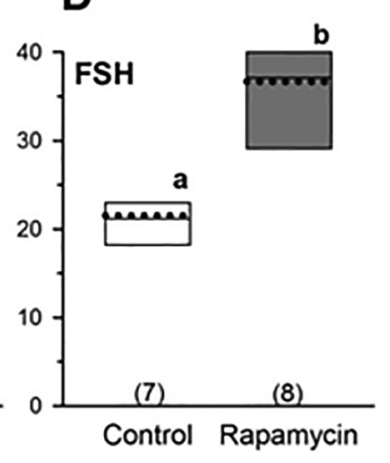

Fig. 8. The mTOR inhibitor rapamycin influences the brain-pituitary-gonadal axis at multiple levels in male Astatotilapia burtoni. (A) Relative levels of the downstream phosphorylated-p70S6 kinase are lower in the whole brain at 48 hrs after intraperitoneal (IP) injection of rapamycin compared to vehicle-injected (control) fish. Inset at top shows representative examples of western blots from whole brains of control vehicle and rapamycin-injected fish. Lower band represents the quantified p-p70S6 isoform, and upper band represents the pp85S6 isoform also detected with this antibody. (B) Relative levels of p-p70S6K are also lower in the testes of IP rapamycin-injected fish compared to controls. (C) Circulating levels of luteinizing hormone (LH) are higher in fish IP injected with rapamycin compared to controls. (D) Circulating levels of follicle stimulating hormone (FSH) are also higher in fish IP injected with rapamycin compared to controls. All measurements were done at $48 \mathrm{hrs}$ after IP rapamycin or vehicle injections. Box boundaries show 25th and 75th percentiles, solid and dotted lines are the median and the mean, respectively. Different letters indicate statistical differences at $\mathrm{p}<0.05$ and sample sizes are shown in parentheses.

induced growth of GnRH1 cells. Similarly, a previous preliminary study in $A$. burtoni showed a trend towards reduced GnRH1 somata size after ICV rapamycin injection in stable dominant males (Kim and Sohn, 2016). Since mtor is expressed throughout the A. burtoni brain, the ICV rapamycin injections may have impacted the GnRH1 neurons either directly or indirectly via upstream circuitry. mTOR signaling is known to control cell growth in a wide variety of cell types, including those processes involved in development and synaptic plasticity in neurons (Jaworski and Sheng, 2006; Sergeeva and Jansen, 2009). Rapamycininduced autophagy also suppresses migration of cultured GN11 cells, implicating mTOR signaling in an immortalized GnRH neuronal cell line (Li et al., 2019). Any reduced hypertrophy of GnRH1 neurons in ascending male $A$. burtoni likely results in delayed up-regulation of the reproductive axis, which has important implications for fertility. To our knowledge, this is the first evidence of mTOR-mediated control of somata size in GnRH1 neurons of any vertebrate.

Transcripts for mtor were also localized to the pituitary of $A$. burtoni by RT-PCR, qPCR, and ISH. Regulation of gonadotrope function by mTOR-signaling pathways is already well-established in mammals. For example, in mammalian-derived gonadotrope cells, mTORC1 plays a role in regulating cap-dependent translation via phosphorylation of both p70S6 kinase and 4E-BP1 (Kim et al., 2014; Oliveira et al., 2017; Stathopoulou et al., 2019). Binding of GnRH1 to its receptors on gonadotrope cells leads to rapid activation of the mTOR signaling cascade, promoting translation initiation and elongation of LH $\beta$ and FSH $\beta$, as well as immediate early genes such as cfos, cjun, and egr1 (Kim et al., 2014). In A. burtoni, we observed higher levels of mtor mRNA at 72-120 hrs after social ascent, a time that also corresponds to increases in pituitary $\operatorname{lh} \beta$ and $f \operatorname{sh} \beta$ mRNA levels (Maruska et al., 2007). Thus, as in mammals, it seems plausible that mTOR-signaling cascades play a role in synthesis and release of LH and FSH in the pituitary of fishes.

When we inhibited the mTOR pathway with IP injections of rapamycin in male A. burtoni, we found increased circulating levels of $\mathrm{LH}$ and FSH. This is consistent with the elevated circulating levels of $\mathrm{LH}$ and FSH (and lower testosterone) commonly observed in clinical studies with patients treated with rapamycin-related compounds (e.g., Sirolimus, Everolimus) (Huyghe et al., 2007; Jesus et al., 2017; Lee et al., 2005; Morrison et al., 2007). In fact, there is ample evidence in the literature for direct effects of systemically-delivered rapamycin on testicular function in mammals, including in humans (Huyghe et al., 2007; Yang et al., 2015). Within the BPG axis, GnRH neurons and LH and FSH production from the pituitary are regulated by a negative feedback loop from sex-steroid hormones released by the gonads. Therefore, rapamycin-induced mTOR suppression at the testes that lowers levels of plasma sex-steroids could reduce this negative feedback to the pituitary and brain, leading to increased LH and FSH release. This reduced negative feedback is the most prevalent hypothesized mechanism in humans. We cannot however, exclude the possibility that IP rapamycin injections have other effects in the body and that non-testis related mechanisms may be involved. Similar effects on plasma LH and FSH between the cichlid and mammals after rapamycin treatment provide further support for conservation of mTOR-signaling function in male reproduction. Future studies are needed, however, to elucidate the exact mechanisms involved in mTOR-mediated regulation of the pituitary and testis.

In the testes of $A$. burtoni, mtor was localized to the germ cells contained within membrane-bound spermatocysts. In mammals, mTOR is found in Sertoli cells and several spermatogonial cell stages (e.g. spermatogonia, spermatocytes) (Fang et al., 2015; Nguyen et al., 2004; Zhao and Fernald, 2005), and was recently shown to be required for spermatogonial differentiation and proliferation in mice and rats (Boyer et al., 2016; Busada et al., 2015; Cao et al., 2019; Yang et al., 2015). Further, mTORC1 is essential to maintain male fertility in mammals (Morrison et al., 2007; Schmelzle and Hall, 2000), and mTOR in the testis has roles in brain-testis-barrier dynamics, meiotic sex chromosome inactivation, and spermatogonial stem cell maintenance, in addition to spermatogenesis (Morrison et al., 2007). In A. burtoni, we found that relative mRNA levels of mtor in the testes were higher at 120 hrs after social ascent, and positively correlated with testicular $f s h$ receptor levels. This $120 \mathrm{hr}$ time point also coincides with higher levels of androgen, estrogen, and glucocorticoid receptors in the testis, as well as augmented spermatogenesis (Maruska and Fernald, 2011). Similarly, in rat testis, mTOR expression also changes with developmental stage in a pattern that parallels sperm production, suggesting that mTOR plays a critical role in mammalian spermatogenesis (Yang et al., 2015). Our data in the cichlid suggest that mTOR pathways may also play a role in spermatogenesis in fishes, providing further evidence for its conserved role in vertebrate male fertility.

In humans, mTOR is also present in Sertoli cells where it regulates nutritional support of spermatogenesis and redox balance (Jesus et al., 2016). It is also possible, therefore, that mTOR in the cichlid testis provides an essential role in Sertoli cell metabolism in support of spermatogenesis, as well as any of the other testicular functions described in mammals (Morrison et al., 2007). mTOR signaling, therefore, may also be involved in gonadotrope and steroid signaling in the fish 
testis, particularly with respect to reproductive competence during social status transitions, a phenomenon that has many relevant similarities to puberty onset in mammals.

Intraperitoneal injections of the mTOR inhibitor rapamycin also reduced testicular activity of the mTOR-signaling pathway (detected by reduced p-p70S6 kinase levels) in A. burtoni. There is accumulating evidence in the literature for a direct effect of rapamycin on the testis (Boyer et al., 2016; Huyghe et al., 2007; Jesus et al., 2017; Oliveira et al., 2017; Weber and Gutmann, 2012; Zhao and Fernald, 2005), and a recent study showed that mTOR preferentially regulates p70S6 kinase rather than translation inhibitory eukaryotic initiation factor 4Ebinding protein-1 (4E-BP1) to promote spermatogonial proliferation (Yang et al., 2015). In mammals, inhibition of mTOR with rapamycin inhibits proliferation of spermatogonia, increases cell apoptosis in the testis, and suppresses phosphorylation of p70S6K to reduce sperm production (Liu et al., 2017). mTOR germ cell-specific knock-out mice also have smaller testes and reduced or blocked spermatogonial differentiation, demonstrating that mTOR acts directly in the testes primarily through mTORC1 in spermatogonia (Skalecka et al., 2016). In addition to regulating spermatogenesis, mTORC1 signaling influences the blood-testis-barrier by modulating organization of the actin cytoskeleton (Li et al., 2019; Li et al., 2018). mTOR function in the testis is of particular clinical interest because inhibitors such as rapamycin and related compounds (Sirolimus, Everolimus) are used in humans as an immunosuppressive drug following some organ transplants, as well as in some cancer treatments, and there is evidence for gonadal dysfunction, defects in spermatogenesis, and reduced fertility in these male patients (Huyghe et al., 2007; Jesus et al., 2017; Morrison et al., 2007). Rapamycin is also being explored and used as a potential treatment for other conditions including Alzheimer's disease (Carosi and Sargeant, 2019), epilepsy (Kim and Lee, 2019), autoimmune disorders (Stevenson et al., 2012), and others, making these fertility effects of even greater importance. While it is possible that the systemic effects of rapamycin may be different in fishes, a recent transcriptome analysis on a zebrafish fibroblast cell line (ZF4) demonstrated conservation of rapamycinmodulated functional pathways between fish and mice (Takahara and Maeda, 2013). Together with our data showing mTOR expression in the testes, and rapamycin-induced effects on the downstream p-p70S6 kinase in the testes, it is likely that mTOR signaling is also essential for spermatogenesis in fishes. Thus, we here provide the first evidence for mTOR involvement of testicular function in fishes, supporting its conserved function in gonadal regulation in male vertebrates.

Since mTOR is a pervasive kinase implicated in numerous cellular processes, it likely has numerous roles in support of male reproduction that we have only begun to explore. Our study examining mTOR in the male reproductive system of a fish is merely a beginning, and we acknowledge several limitations of our study that require future investigation. For example, while we show changes in mtor mRNA levels in the pituitary and testis as males transition in social rank, the functional implications of fluctuations in mRNA is not known. One simple interpretation is that more transcripts are available for translation and incorporation into the active mTORC $1 / 2$ complexes, but quantification of activated signaling cascades is needed to test this hypothesis. Further, it is important to note that regulation of the BPG axis is complex and involves feedback loops that influence upstream processes, so the exact location and mechanism of our observed IP rapamycin effects cannot be determined. However, these injections resemble the systemic effects seen in patients treated with rapamycin (e.g. increased LH and FSH levels, reduced testicular activity), suggesting it may be similar to the direct impacts on the testis demonstrated in mammals (Morrison et al., 2007). It is also unknown whether or not rapamycin delivered via IP injection influences the GnRH1 neurons directly, although we did observe inhibition of mTOR signaling in the brain and there is evidence in mammals that rapamycin does cross the blood brain barrier (Banerjee et al., 2011). The fact that our ICV rapamycin injections reduced the canonical hypertrophy of GnRH1 neurons during social ascent raises the possibility that IP rapamycin may also inhibit GnRH1 neuron activity, thereby reducing LH and FSH release from the pituitary. In contrast, however, we actually observed increased circulating LH and FSH levels after IP rapamycin injection in dominant males. ICV injections were done in ascending males while IP injections were done in stable dominant males, so differences may be due to male physiological state or injection location. Alternatively, IP injection allowed rapamycin to target many tissue sites and regulatory pathways, possibly having a greater effect on reducing the negative feedback to the brain and pituitary than on reduced GnRH1 activity, resulting in higher pituitary gonadotropin release in these reproductively active males. Despite these current limitations, our results support the utility of $A$. burtoni as a well-suited model to further explore the potential role of mTOR signaling in male reproductive function of fishes.

\section{Conclusions}

mTOR signaling pathways are ubiquitous in eukaryotic organisms, with many crucial functions in cellular growth, metabolism, and physiology demonstrated in a wide range of different cell types. It is becoming clearer that mTOR is a complex integrator of a plethora of physiological signals, and along with these signals, plays a role in several coordinated cellular processes that lead to important functional consequences for the cell, tissue, organ, and ultimately the whole animal. While mTOR's role in regulation of the neuroendocrine reproductive axis was previously shown in mammals (primarily in females), our data reveal its involvement in the brain-pituitary-gonadal axis of male fishes. mTOR, or components of its downstream signaling pathways, are expressed in GnRH1 neurons of the brain, in the pituitary, and in the testis of the cichlid fish A. burtoni. We show sociallyinduced differences in mTOR activity between reproductively-suppressed subordinate males, and males that were given an opportunity to rise in rank to become reproductively-active dominant males, a transition similar to puberty in mammals. Treatment with the mTOR inhibitor rapamycin also influenced mTOR pathway activity in the brain, pituitary, and testes, including inhibition of the canonical socially-induced GnRH1 neuron hypertrophy. These data suggest that the rapamycin-sensitive mTORC1 complex plays a role in the physiological function of GnRH1 neurons, pituitary gonadotropes, and germ cells of the testis. Our integrative analysis that extends the discovered function of mTOR to the reproductive axis in males of a non-mammal provides support for the hypothesis that mTOR signaling is a conserved and crucial mechanism involved in male reproductive competence across vertebrates.

\section{Funding}

This work was supported in part by National Institutes of Health (NIH no. F32NS061431 to KPM and NIH no. NS034950 to RDF), National Science Foundation (IOS-1456004 and IOS-1456558 to KPM), startup funds from the College of Science and Department of Biological Sciences at Louisiana State University (KPM), and the Basic Science Research Program through the National Research Foundation (NRF) of Korea funded by the Ministry of Education (2012R1A1A2044506 to YCS).

\section{Acknowledgements}

We thank Geet Chakraborty, Helen McCurdy, David Roberts, and Julie Butler for experimental assistance, the Berta Levavi-Sivan lab (Hebrew University, Israel) for LH and FSH ELISAs, and the Walbot lab (Stanford University) for use of the Zeiss PALM LCM system. 


\section{References}

Banerjee, S., Gianino, S.M., Gao, F., Christians, U., Gutmann, D.H., 2011. Interpreting mammalian target of rapamycin and cell growth inhibition in a genetically engineered mouse model of Nf1-deficient astrocytes. Mol. Cancer Ther. 10, 279-291.

Boyer, A., Girard, M., Thimmanahalli, D.S., Levasseur, A., Celeste, C., Paquet, M., et al., 2016. mTOR regulates gap junction alpha-1 protein trafficking in sertoli cells and is required for the maintenance of spermatogenesis in mice. Biol. Reprod. 95, 13.

Bryant, A.S., Greenwood, A.K., Juntti, S.A., Byrne, A.E., Fernald, R.D., 2016. Dopaminergic inhibition of gonadotropin-releasing hormone neurons in the cichlid fish Astatotilapia burtoni. J. Exp. Biol. 219, 3861-3865.

Burmeister, S.S., Jarvis, E.D., Fernald, R.D., 2005. Rapid behavioral and genomic responses to social opportunity. PLoS Biol. 3, e363.

Burmeister, S.S., Wilczynski, W., 2005. Social signals regulate gonadotropin-releasing hormone neurons in the green treefrog. Brain Behav. Evol. 65, 26-32.

Busada, J.T., Niedenberger, B.A., Velte, E.K., Keiper, B.D., Geyer, C.B., 2015. Mammalian target of rapamycin complex 1 (mTORC1) Is required for mouse spermatogonial differentiation in vivo. Dev. Biol. 407, 90-102.

Butler, J.M., Maruska, K.P., 2016. The mechanosensory lateral line system mediates activation of socially-relevant brain regions during territorial interactions. Front. Behav. Neurosci. 10, 93.

Butler, J.M., Maruska, K.P., 2019. Expression of tachykinin3 and related reproductive markers in the brain of the African cichlid fish Astatotilapia burtoni. J. Comp. Neurol. 527, 1210-1227.

Cao, J., Lin, Z.B., Tong, M.H., Zhang, Y.L., Li, Y.P., Zhou, Y.C., 2019. Mechanistic target of rapamycin kinase (Mtor) is required for spermatogonial proliferation and differentiation in mice. Asian J Androl.

Carosi, J.M., Sargeant, T.J., 2019. Rapamycin and Alzheimer disease: a double-edged sword? Autophagy 1-3.

Chiang, G.G., Abraham, R.T., 2005. Phosphorylation of mammalian target of rapamycin (mTOR) at Ser-2448 is mediated by p70S6 kinase. J. Biol. Chem. 280, 25485-25490.

Davis, M.R., Fernald, R.D., 1990. Social control of neuronal soma size. J. Neurobiol. 21, 1180-1188.

Dellovade, T.L., Rissman, E.F., 1994. Gonadotropin-releasing hormone-immunoreactive cell numbers change in response to social interactions. Endocrinology 134, 2189-2197.

Ding, Y., Sun, X., Huang, W., Hoage, T., Redfield, M., Kushwaha, S., et al., 2011. Haploinsufficiency of target of rapamycin attenuates cardiomyopathies in adult zebrafish. Circ. Res. 109, 658-669.

Fang, J., Li, D.H., Yu, X.Q., Lv, M.Q., Bai, L.Z., Du, L.Z., et al., 2015. Formaldehyde exposure inhibits the expression of mammalian target of rapamycin in rat testis. Toxicol. Ind. Health.

Fernald, R.D., Maruska, K.P., 2012. Social information changes the brain. Proc. Natl. Acad. Sci. USA 109 (Suppl 2), 17194-17199.

Flanagan, C.A., Chen, C.C., Coetsee, M., Mamputha, S., Whitlock, K.E., Bredenkamp, N., et al., 2007. Expression, structure, function, and evolution of gonadotropin-releasing hormone (GnRH) receptors GnRH-R1SHS and GnRH-R2PEY in the teleost Astatotilapia burtoni. Endocrinology 148, 5060-5071.

Francis, R.C., Soma, K., Fernald, R.D., 1993. Social regulation of the brain-pituitary-gonadal axis. PNAS 90, 7794-7798.

Greenwood, A.K., Fernald, R.D., 2004. Social regulation of the electrical properties of gonadotropin-releasing hormone neurons in a cichlid fish (Astatotilapia burtoni). Biol. Reprod. 71, 909-918.

Grone, B.P., Maruska, K.P., 2015. A second corticotropin-releasing hormone gene (CRH2) is conserved across vertebrate classes and expressed in the hindbrain of a basal neopterygian fish, the spotted gar (Lepisosteus oculatus). J. Comp. Neurol. 523, 1125-1143.

Grone, B.P., Maruska, K.P., 2015. Divergent evolution of two corticotropin-releasing hormone (CRH) genes in teleost fishes. Front. Neurosci. 9, 365.

Hirose, K., Shiomi, T., Hozumi, S., Kikuchi, Y., 2014. Mechanistic target of rapamycin complex 1 signaling regulates cell proliferation, cell survival, and differentiation in regenerating zebrafish fins. BMC Dev. Biol. 14, 42.

Huyghe, E., Zairi, A., Nohra, J., Kamar, N., Plante, P., Rostaing, L., 2007. Gonadal impact of target of rapamycin inhibitors (sirolimus and everolimus) in male patients: an overview. Transplant Int. 20, 305-311.

Jacinto, E., Loewith, R., Schmidt, A., Lin, S., Ruegg, M.A., Hall, A., et al., 2004. Mammalian TOR complex 2 controls the actin cytoskeleton and is rapamycin insensitive. Nat. Cell Biol. 6, 1122-1128.

Jaworski, J., Sheng, M., 2006. The growing role of mTOR in neuronal development and plasticity. Mol. Neurobiol. 34, 205-219.

Jesus, T.T., Oliveira, P.F., Silva, J., Barros, A., Ferreira, R., Sousa, M., et al., 2016. Mammalian target of rapamycin controls glucose consumption and redox balance in human Sertoli cells. Fertility Sterility 105, 825-833 e823.

Jesus, T.T., Oliveira, P.F., Sousa, M., Cheng, C.Y., Alves, M.G., 2017. Mammalian target of rapamycin (mTOR): a central regulator of male fertility? Crit. Rev. Biochem. Mol. Biol. 52, 235-253.

Jiang, J., Feng, L., Liu, Y., Jiang, W.D., Hu, K., Li, S.H., et al., 2013. Mechanistic target of rapamycin in common carp: cDNA cloning, characterization, and tissue expression. Gene 512, 566-572.

Kim, T., Do, M.H., Lawson, M.A., 2014. Translational control of gene expression in the gonadotrope. Mol. Cell. Endocrinol. 385, 78-87.

Kim, J.K., Lee, J.H., 2019. Mechanistic target of rapamycin pathway in epileptic disorders. J. Korean Neurosurg. Soc. 62, 272-287.

Kim, T.H., Sohn, Y.C., 2016. Changes of Sexual Behaviors in Rapamycin-injected Cichlid Fish Astatotilapia burtoni Males. Devel. Reproduction 20, 267-274.
Kriegsfeld, L.J., Nelson, R.J., 1999. Photoperiod affects the gonadotropin-releasing hormone neuronal system of male prairie voles (Microtus ochrogaster). Neuroendocrinology 69, 238-244.

Laplante, M., Sabatini, D.M., 2009. mTOR signaling at a glance. J. Cell Sci. 122, 3589-3594.

Lee, S., Coco, M., Greenstein, S.M., Schechner, R.S., Tellis, V.A., Glicklich, D.G., 2005. The effect of sirolimus on sex hormone levels of male renal transplant recipients. Clin. Transplant. 19, 162-167.

Li, F., Li, D., Liu, H., Cao, B., Jiang, F., Chen, D., et al., 2019. RNF216 regulates the migration of immortalized GnRH neurons by suppressing beclin1-mediated autophagy. Front. Endocrinol.

Li, L.X., Wu, S.W., Yan, M., Lian, Q.Q., Ge, R.S., Cheng, C.Y., 2019. Regulation of bloodtestis barrier dynamics by the mTORC1/rpS6 signaling complex: An in vitro study. Asian J Androl.

Li, S., Yan, M., Chen, H., Jesus, T., Lee, W., Xiao, X., et al., 2018. mTORC1/rpS6 regulates blood-testis barrier dynamics and spermatogenic function in the testis in vivo. Am. J. Physiol. Endocrinol. Metab. 314, E174-E190.

Liu, S., Huang, L., Geng, Y., He, J., Chen, X., Xu, H., et al., 2017. Rapamycin inhibits spermatogenesis by changing the autophagy status through suppressing mechanistic target of rapamycin-p70S6 kinase in male rats. Mol. Med. Rep. 16, 4029-4037.

Ma, Y., Juntti, S.A., Hu, C.K., Huguenard, J.R., Fernald, R.D., 2015. Electrical synapses connect a network of gonadotropin releasing hormone neurons in a cichlid fish. Proc. Natl. Acad. Sci. USA 112, 3805-3810.

MacDougall-Shackleton, S.A., Stevenson, T.J., Watts, H.E., Pereyra, M.E., Hahn, T.P., 2009. The evolution of photoperiod response systems and seasonal GnRH1 plasticity in birds. Integr. Comp. Biol. 49, 580-589.

Makky, K., Mayer, A.N., 2007. Zebrafish Offers New Perspective on Developmental Role of TOR Signaling. Organogenesis. 3, 67-69.

Makky, K., Tekiela, J., Mayer, A.N., 2007. Target of rapamycin (TOR) signaling controls epithelial morphogenesis in the vertebrate intestine. Dev. Biol. 303, 501-513.

Maruska, K.P., 2009. Sex and temporal variations of the vasotocin neuronal system in the damselfish brain. Gen. Comp. Endocrinol. 160, 194-204.

Maruska, K.P., 2014. Social regulation of reproduction in male cichlid fishes. Gen. Comp. Endocrinol. 207, 2-12.

Maruska, K.P., 2015. Social Transitions Cause Rapid Behavioral and Neuroendocrine Changes. Integr. Comp. Biol.

Maruska, K.P., Fernald, R.D., 2010. Behavioral and physiological plasticity: rapid changes during social ascent in an African cichlid fish. Horm. Behav. 58, 230-240.

Maruska, K.P., Fernald, R.D., 2011. Plasticity of the reproductive axis caused by social status change in an African cichlid fish: II. testicular gene expression and spermatogenesis. Endocrinology 152, 291-302.

Maruska, K.P., Fernald, R.D., 2013. Social regulation of male reproductive plasticity in an african cichlid fish. Integr. Comp. Biol. 207, 2-12.

Maruska, K.P., Fernald, R.D., 2014. Social regulation of gene expression in the African cichlid fish Astatotilapia burtoni. In: Canli, T. (Ed.), Handbook of Molecular Psychology. Oxford University Press, New York, N.Y., pp. 52-78.

Maruska, K.P., Fernald, R.D., 2018. Astatotilapia burtoni: a model system for analyzing the neurobiology of behavior. ACS Chem. Neurosci.

Maruska, K.P., Tricas, T.C., 2011. Gonadotropin-releasing hormone (GnRH) modulates auditory processing in the fish brain. Horm. Behav. 59, 451-464.

Maruska, K.P., Mizobe, M.H., Tricas, T.C., 2007. Sex and seasonal co-variation of arginine vasotocin (AVT) and gonadotropin-releasing hormone $(\mathrm{GnRH})$ neurons in the brain of the halfspotted goby. Comp. Biochem. Physiol. A: Mol. Integr. Physiol. 147, 129-144.

Maruska, K.P., Levavi-Sivan, B., Biran, J., Fernald, R.D., 2011. Plasticity of the reproductive axis caused by social status change in an African cichlid fish: I. Pituitary gonadotropins. Endocrinology 152, 281-290.

Maruska, K.P., Becker, L., Neboori, A., Zhang, A., Fernald, R.D., 2012. Social transitions cause rapid behavioral, endocrine, and transcriptional changes in the brain of an African cichlid fish. Soc. for Neurosci Abstr., New Orleans, LA. p. 385.301.

Maruska, K.P., Zhang, A., Neboori, A., Fernald, R.D., 2012. Social opportunity causes rapid transcriptional changes in the social behaviour network of the brain in an African cichlid fish. J. Neuroendocrinol.

Maruska, K.P., Zhang, A., Neboori, A., Fernald, R.D., 2013. Social opportunity causes rapid transcriptional changes in the social behavior network of the brain in an African cichlid fish. J. Neuroendocrinol. 25, 145-157.

Maruska, K.P., Butler, J.M., Field, K.E., Porter, D.T., 2017. Localization of glutamatergic, GABAergic, and cholinergic neurons in the brain of the African cichlid fish, Astatotilapia burtoni. J. Comp. Neurol. 525, 610-638.

Moreira, B.P., Oliveira, P.F., Alves, M.G., 2019. Molecular mechanisms controlled by mTOR in male reproductive system. Int. J. Mol. Sci. 20.

Morrison, C.D., Xi, X., White, C.L., Ye, J., Martin, R.J., 2007. Amino acids inhibit Agrp gene expression via an mTOR-dependent mechanism. Am. J. Physiol. Endocrinol. Metab. 293, E165-E171.

Mukherjee, A., Koli, S., Reddy, K.V., 2015. Rapamycin (Sirolimus) alters mechanistic target of rapamycin pathway regulation and microRNA expression in mouse meiotic spermatocytes. Andrology. 3, 979-990.

Nguyen, K.A., Santos, S.J., Kreidel, M.K., Diaz, A.L., Rey, R., Lawson, M.A., 2004. Acute regulation of translation initiation by gonadotropin-releasing hormone in the gonadotrope cell line LbetaT2. Mol. Endocrinol. 18, 1301-1312.

Oliveira, P.F., Cheng, C.Y., Alves, M.G., 2017. Emerging role for mammalian target of rapamycin in male fertility. Trends Endocrinol. Metab. 28, 165-167.

Penney, C.C., Volkoff, H., 2014. Peripheral injections of cholecystokinin, apelin, ghrelin and orexin in cavefish (Astyanax fasciatus mexicanus): effects on feeding and on the brain expression levels of tyrosine hydroxylase, mechanistic target of rapamycin and appetite-related hormones. Gen. Comp. Endocrinol. 196, 34-40.

Pullen, N., Thomas, G., 1997. The modular phosphorylation and activation of p70s6k. 
FEBS Lett. 410, 78-82.

Roa, J., Garcia-Galiano, D., Varela, L., Sanchez-Garrido, M.A., Pineda, R., Castellano, J.M., et al., 2009. The mammalian target of rapamycin as novel central regulator of puberty onset via modulation of hypothalamic Kiss1 system. Endocrinology 150, 5016-5026.

Roa, J., Garcia-Galiano, D., Castellano, J.M., Gaytan, F., Pinilla, L., Tena-Sempere, M., 2010. Metabolic control of puberty onset: new players, new mechanisms. Mol. Cell. Endocrinol. 324, 87-94.

Roa, J., Tena-Sempere, M., 2014 Connecting metabolism and reproduction: Roles of central energy sensors and key molecular mediators. Mol. Cell. Endocrinol. 397, 4-14.

Scanlon, M.D., Greenwood, A.K., Fernald, R.D., 2003. Dendritic plasticity in gonadotropin-releasing hormone neurons following changes in reproductive status. Soc. Neurosci. p. 828.820

Schell, C., Kretz, O., Liang, W., Kiefer, B., Schneider, S., Sellung, D., et al., 2016. The rapamycin-sensitive complex of mammalian target of rapamycin is essential to maintain male fertility. Am. J. Pathol. 186, 324-336.

Schmelzle, T., Hall, M.N., 2000. TOR, a central controller of cell growth. Cell 103, 253-262.

Sergeeva, A., Jansen, H.T., 2009. Neuroanatomical plasticity in the gonadotropin-releasing hormone system of the ewe: seasonal variation in glutamatergic and gammaaminobutyric acidergic afferents. J. Comp. Neurol. 515, 615-628.

Serra, N.D., Velte, E.K., Niedenberger, B.A., Kirsanov, O., Geyer, C.B., 2017. Cell-autonomous requirement for mammalian target of rapamycin (Mtor) in spermatogonial proliferation and differentiation in the mousedagger. Biol. Reprod. 96, 816-828.

Skalecka, A., Liszewska, E., Bilinski, R., Gkogkas, C., Khoutorsky, A., Malik, A.R., et al., 2016. mTOR kinase is needed for the development and stabilization of dendritic arbors in newly born olfactory bulb neurons. Dev Neurobiol.

Sosnowski, R., Mellon, P.L., Lawson, M.A., 2000. Activation of translation in pituitary gonadotrope cells by gonadotropin-releasing hormone. Mol. Endocrinol. 14,
$1811-1819$.

Stathopoulou, C., Nikoleri, D., Bertsias, G., 2019. Immunometabolism: an overview and therapeutic prospects in autoimmune diseases. Immunotherapy. 11, 813-829.

Stevenson, T.J., Hahn, T.P., Ball, G.F., 2012. Variation in gonadotrophin-releasing hormone-1 gene expression in the preoptic area predicts transitions in seasonal reproductive state. J. Neuroendocrinol. 24, 267-274.

Stevenson, T.J., Hahn, T.P., MacDougall-Shackleton, S.A., Ball, G.F., 2012. Gonadotropinreleasing hormone plasticity: a comparative perspective. Front. Neuroendocrinol. 33 287-300.

Sucularli, C., Shehwana, H., Kuscu, C., Dungul, D.C., Ozdag, H., Konu, O., 2016. Functionally conserved effects of rapamycin exposure on zebrafish. Mol. Med. Rep. 13, 4421-4430.

Takahara, T., Maeda, T., 2013. Evolutionarily conserved regulation of TOR signalling. J. Biochem. 154, 1-10.

van Dam, T.J.P., Zwartkruis, F.J.T., Bos, J.L., Snel, B., 2011. Evolution of the TOR pathway. J. Mol. Evol. 73, 209-220.

Weber, J.D., Gutmann, D.H., 2012. Deconvoluting mTOR biology. Cell Cycle 11, 236-248.

Xu, H., Shen, L., Chen, X., Ding, Y., He, J., Zhu, J., et al., 2016. mTOR/P70S6K promotes spermatogonia proliferation and spermatogenesis in Sprague Dawley rats. Reprod. Biomed. Online 32, 207-217.

Yang, W.R., Wang, Y., Zhang, J.J., Zhang, J.H., Lu, C., Wang, X.Z., 2015. mTOR is involved in 17beta-estradiol-induced, cultured immature boar Sertoli cell proliferation via regulating the expression of SKP2, CCND1, and CCNE1. Mol. Reprod. Dev. 82, 305-314.

Zhao, S., Fernald, R.D., 2005. Comprehensive algorithm for quantitative real-time polymerase chain reaction. J. Comput. Biol. 12, 1047-1064.

Zuber, J., Anglicheau, D., Elie, C., Bererhi, L., Timsit, M.O., Mamzer-Bruneel, M.F., et al., 2008. Sirolimus may reduce fertility in male renal transplant recipients. Am. J. Transpl. 8, 1471-1479. 